\title{
GROUP DECISION-MAKING MODELS FOR VENTURE CAPITALISTS: THE PROMETHEE WITH HESITANT FUZZY LINGUISTIC INFORMATION
}

\author{
Xiaoli TIAN ${ }^{1}$, Zeshui $\mathrm{XU}^{2 *}$, Jing $\mathrm{GU}^{3}$ \\ ${ }^{1}$ School of Business Administration, Southwestern University of Finance and Economics, \\ Chengdu, 610074, China \\ ${ }^{2}$ Business School, Sichuan University, Chengdu, 610065, China \\ ${ }^{3}$ School of Economics, Sichuan University, Chengdu,610065, China
}

Received 05 January 2018; accepted 16 December 2018

\begin{abstract}
Venture capitalists (VCs) have long been preoccupied by the issue of selecting a promising start-up firm, whereas, ranking the available start-up firms is an effective way to solve this issue. In this paper, the PROMETHEE is chosen to be the fundamental ranking method. Also, the hesitant fuzzy linguistic term set is a suitable tool to simulate VCs' evaluation information. Additionally, as the deepening of social division of labor and specialization of individuals, group decision making is famous for improving decision-making quality. Moreover, in the decision-making process, VCs exhibit behavioral characteristics which is depicted well by prospect theory that VCs are risk averse for gains and risk seeking for losses and rely on the transformed probability to make their decisions rather than unidimensional probability. Thus, a group prospect PROMETHEE with hesitant fuzzy linguistic information is constructed for VCs to make a better decision. Then, the proposed method is applied to rank start-up firms and the comparative analyses are made as well. It confirms that the group prospect PROMETHEE is better in describing the common behavioral characteristics of VCs and in enhancing the quality of evaluation.
\end{abstract}

Keywords: group decision making, PROMETHEE, prospect theory, hesitant fuzzy linguistic information, venture capital.

JEL Classification: D81, G41.

\section{Introduction}

In order to transform economic structure and improve the graduates' employment rate, the mass entrepreneurship and innovation is highlighted by the government of China. Undoubtedly, numerous start-up firms born in entrepreneurship and innovation are of new technology, high risk and potential high revenue, which need the help of capital. However, venture capital (VC), as the distinctive type of financing start-up firms for the sake of obtaining

*Corresponding author. E-mail: xuzeshui@263.net

Copyright (C) 2019 The Author(s). Published by VGTU Press

This is an Open Access article distributed under the terms of the Creative Commons Attribution License (http://creativecommons. org/licenses/by/4.0/), which permits unrestricted use, distribution, and reproduction in any medium, provided the original author and source are credited. 
the above-average revenue in the circumstance of high uncertainty, becomes a popular tool for start-up firms to acquire capital and has already caught exceptional prominence in the society.

The VC is usually operated by venture capitalists (VCs) and there are some steps for the VCs to successfully fund a start-up firm. Nevertheless, how to find a promising start-up firm has been the key step for the success of VC. Hence, the detailed assessments for the optional start-up firms appear to be a particularly important issue. In the process of detailed assessments, there have been some criteria used by the VCs to make an overall judgement, such as the comprehensive quality of management team, the market opportunity, the product/service differentiation and the financial situation.

It is easy for the VCs to evaluate those criteria as linguistic terms because of the qualitative characteristics of those criteria and the universality of linguistic expressions in daily life. For example, when a venture capitalist is asked to evaluate the business experience of management team, he/she tends to use the sentence "the business experience of management team is rich" or "the management team is lack of business experience" to express his/ her perception. It is obvious that the criteria such as the ability of the management team, the market potential, the quality of product or service, etc. are qualitative, which is hard for the VCs to estimate them as crisp numbers. Thus, linguistic expressions should be introduced to simulate such situations.

In addition, sometimes, the VCs are hesitant in linguistic expressions, especially in the highly uncertain decision-making situations. For instance, the bike-share programs, such as 'ofo', 'mobike', etc., have recently caught much attention from the VCs in China. When a venture capitalist is asked to evaluate the service quality of 'mobike', he/she is more likely to express his/her true feelings with the sentence like "from the aspect of convenience, 'mobike' is at least good". Here, the "at least good" means that the VCs are hesitant in "good" or "very good". Moreover, the sentence "from the frequency of utilization, 'mobike' is very high" has only one linguistic term which is a special situation for hesitant fuzzy linguistic expression. Therefore, hesitant fuzzy linguistic term sets (HFLTSs) (Rodríguez, Martínez, \& Herrera, 2012) are suitable for depicting the above linguistic expression situations under highly uncertain environment. Furthermore, Rodríguez et al. (2012) comprehensively explained how the context free grammar generates linguistic expressions. Since it is not the key point of this paper to present the context-free grammar, we do not explain this detailed process here (please refer to Rodríguez et al., 2012 for more details).

The uncertain environment creates psychological discomfort for the VCs. In order to reduce such discomfort produced by uncertainty, the VCs will take the advantage of decision-making model to provide a reference for them to make their choices. Furthermore, the choice under uncertainty is of bounded rationality instead of complete rationality, whereas, the prospect theory (PT) (Kahneman \& Tversky, 1979) is an optional tool for the individuals to describe their choices with bounded rationality under uncertain circumstance through the value function and to simulate their perceived probability by virtue of the transformed weighting function. As the founder of PT emphasized, the individuals make their decisions with bounded rationality and they tend to be risk averse for gains and risk seeking for losses when the decision-making situation is uncertain. Such risk attitudes for gains and losses have 
been particularly highlighted among the VCs who prefer to gain extra high revenue and bear high risk. Thus, introducing the PT and the hesitant fuzzy linguistic information into the VC field for the VCs to make a more reasonable decision is not only meaningful for the VCs' decision-making but also beneficial to the VC corporate' performance.

The decision-making of the VCs depends on several criteria, so it is a multi-criteria decision-making (MCDM) problem as well. However, the outranking method is one of the effective way to solve the MCDM problem. Furthermore, the PROMETHEE (Preference Ranking Organization METHod for Enrichment Evaluations) is one of the best-known ranking methods (Kahraman, Onar, \& Oztaysi, 2015). It reflects more information about the alternative through the net outranking flow (including both the positive outranking flow and the negative outranking flow). For an alternative, both the positive outranking flow and the negative outranking flow to the other alternatives are considered in the PROMETHEE. Such a global opinion comprehensively embodies the relative preference of alternatives well. In addition, it is widely used in decision sciences and social sciences (Kahraman et al., 2015). Hence, the PROMETHEE is totally adequate to select the promising start-up firm for VCs. Also, uncertainty is the fundamental characteristic of the $\mathrm{VC}$ and it exists in the whole decision-making process. Moreover, group decision making is an effective way to aggregate the opinions of each decision maker, which can enable the VCs to make full use of their advantages and to make up for their shortcomings in individual decision-making. Therefore, considering the evaluation behavior of the VCs under uncertain VC environment and the necessity of ranking those available start-up firms, two group decision-making methods with hesitant fuzzy linguistic information have been constructed and used to analyze the decision-making problem of VCs/VC firms in this paper.

The main contributions of this paper can be summarized as follows: (1) The detailed steps of both the group prospect PROMETHEE (GP-PROMETHEE) and the group PROMETHEE (G-PROMETHEE) are structured under hesitant fuzzy linguistic circumstance in this paper. (2) The group decision making, which not only makes full use of each venture capitalist's advantages but also makes up for each venture capitalist's shortcomings in individual decision-making, is considered in the proposed methods. (3) The hesitant fuzzy linguistic information has been introduced to portray the qualitative evaluation information of the VCs for the first time. (4) The risk aversion for gains and risk seeking for losses of the VCs have been fully considered in the GP-PROMETHEE. It is the first time to integrate decision makers' psychological characteristics into the PROMETHEE.

The rest of this paper is organized as follows: Section 1 shows the current researches from three aspects: (1) The existing decision-making models used by the VCs; (2) The decisionmaking methods on the basis of the PROMETHEE and its extensions; (3) The application fields of the PROMETHEE and its extensions. In order to complete the procedures of the proposed methods, the basic concepts such as the HFLTSs and the PT are introduced in Section 2. Then, the main focus of this paper is given in Section 3 because the decision-making methods including the GP-PROMETHEE and the G-PROMETHEE are constructed under hesitant fuzzy linguistic circumstance. Also, the detailed steps and the virtual procedure of the GP-PROMETHEE are narrated here. In Section 4, an illustrative example is used to show the feasibility of the proposed methods. Also, to further investigate the better results of 
the GP-PROMETHEE, the comparative analyses are conducted in this section through the comparison of GP-PROMETHEE and G-PROMETHEE and the comparison of GP-PROMETHEE and TODIM (TOmada de Decisão Iterativa Multicritério). Finally, the paper ends with some conclusions.

\section{Literature reviews}

In this section, a series of literature reviews are listed to show the existing researches about the decision-making of VCs and about the PROMETHEE.

\subsection{Existing decision-making models used by the VCs}

For the sake of constructing an appropriate decision-making model for the VCs, it is important for us to know the existing decision-making models used by the VCs. Several decisionmaking technologies have been introduced for the VCs to select a promising start-up firm. For example, the DCF (discounted cash flow) was used to choose a promising start-up firm based on the prediction of future value and discount rate (Zhao, 2009). However, such prediction may be wrong and increases the probability of inaccurate decision-making. The AHP (Analytical Hierarchy Process) (Gannon, Hogan, \& Olson, 2015) was employed as a tool in decision-making. Though the AHP can integrate both qualitative and quantitative evaluation information, the independence of decision-making criteria is the basic assumption of the AHP. Hence, the situation that the decision-making criteria are dependent is ignored. Then, the ANP (Analytic Network Process) (Wiratno, Latiffianti, \& Wirawan, 2015) fully considering such dependence was applied in selecting start-up firm. But the ranking result from the ANP is not stable when one or few start-up firms are removed from the optional start-up firms. Also, when using the ranking results from the ANP, an additional evaluation and analysis is essential before the final decision-making. This leads the decision-making to be more complex and biased.

Furthermore, with the satisfaction function, a GPM (Goal Programming model) and a stochastic GPM were constructed as decision aid for the VCs (Colapinto \& Torre, 2015). Sometimes, the GPMs with preset objective and constraint condition could not find an optimal start-up firm. Most importantly, all those models have taken the evaluation information as real numbers, and none of them considers the uncertain characteristic of the VC. Thus, the fuzzy optimal model (Zhang, 2012), the fuzzy GPM (Aouni, Colapinto, \& Torre, 2014) were provided to depict uncertainty. Even if the uncertainty has been integrated into those models, the qualitative evaluation information cannot be well portrayed in those models either, especially the linguistic information. Then, considering such qualitative linguistic information, an appraisal model was developed by Zhou (2012) on the basis of high-tech outcome transformation, whereas, it concentrated on the comprehensive evaluation of each alternative but it neglected the relative preference between the alternatives and the bounded rationality of the VCs in the decision-making process. Hence, in this paper, we are dedicated to constructing a linguistic decision-making model including both the relative preference between the alternatives which is well realized through the PROMETHEE and the risk attitudes of the VCs under the bounded rationality that is depicted by the PT. 
As a widely used MCDM method, the PROMETHEE adopts pairwise comparisons of start-up firms to find a promising one through a preference function under each criterion. With the preference function, the strength of preference for one start-up firm to another is calculated. Because of the complex and uncertain investment environment, not all the start-up firms in the market are accessed by the VCs and selecting a promising start-up firm via direct evaluation information obtained from the VCs becomes unsuitable and difficult. On the contrary, using relative preference within the accessed start-up firms to measure the relative advantage of each start-up firm is more reasonable and valuable. Furthermore, for a start-up firm, the net outranking flow (both positive and negative) to the other start-up firms are considered in the PROMETHEE. Such a global opinion embodies the relative preference of start-up firms well, and it could not be realized depending on other MCDM method.

\subsection{PROMETHEE and its extension under fuzzy circumstance}

The PROMETHEE is an outranking method based on the relative preferences that was first proposed by Brans (1982), and then it was extended (Brans \& Vincke, 1985). The evaluation information in the conventional PROMETHEE has been expressed as crisp numbers, which is inconsistent with the complexity of objective affairs. In order to improve the effectiveness of the PROMETHEE, fuzzy set was introduced into the PROMETHEE to portray the uncertain context (Mateo, 2012; W. X. Li \& B. Y. Li, 2010; Chen, 2014). Taking both the support and opposition information into account, the intuitionistic fuzzy PROMETHEE (Liao $\& \mathrm{Xu}, 2014$ ) and the interval-valued intuitionistic fuzzy PROMETHEE (Chen, 2015) were developed, but they could not deal with the qualitative linguistic information. Therefore, considering that some criteria are hard for the decision makers to evaluate by crisp numbers or by intuitionistic fuzzy ways, the PROMETHEE model with fuzzy linguistic information was built to handle qualitative decision-making situations (Chen, Hung, \& Cheng, 2011a; Liao, Wang, \& Liu, 2016). Later, based on the decision-making situation that the decision maker may be hesitant in several possible values, the hesitant fuzzy information was introduced to the PROMETHEE with the purpose of describing hesitant situations (Mahmoudi, Sadi-Nezhad, Makui, \& Vakili, 2016). Since then, more explorations were made such as the fuzzy AHP PROMETHEE (Peko, Gjeldum, \& Bilić, 2018; Samanlioglu \& Ayağ, 2017), the combination of ANP and PROMETHEE (Samanlioglu \& Ayağ, 2016), the fuzzy mathematical programming PROMETHEE (Jiménez, 2005).

Although the PROMETHEE has been extended in various fuzzy circumstances, the PROMETHEE under hesitant fuzzy linguistic circumstance has been ignored until now, especially considering the risk attitudes in the decision-making model. While, along with the development of fuzzy theory, the hesitant fuzzy linguistic information is an effective way to handle the situation in which the decision makers are hesitant to express their linguistic evaluation information. Thereby, it is widespread in general qualitative decision-making problem. Thus, the combination of the HTLTSs and the PROMETHEE with risk attitudes is in urgent need of construction. 


\subsection{Application of the PROMETHEE and its extension}

The PROMETHEE was utilized to rank and select business projects by Brans, Vincke, and Mareschal (1986). Without question, it is of vital importance to select the evaluation criteria which affect the quality of decision-making in the whole process. Bouri, Martel, and Chabchoub (2002) adopted the PROMETHEE and the criteria such as return, risk, liquidity, size and the earning price ratio to obtain an attractive portfolio in the Tunisian Stock Market. While in the decision-making process, it is important for the decision makers to determine the weights of those criteria as well. Thus, Babic and Plazibat (1998) combined the AHP with the PROMETHEE to investigate the promising firm. By virtue of the modified PROMETHEE, Govindan, Kadziński, and Sivakumar (2017) solve the green supplier selection problem.

Because the high degree of uncertainty lies in the decision-making process, the fuzzy PROMETHEE was applied to solve the problem of selecting start-up firm for public VC by Afful-Dadzie, Oplatková, and Nabareseh (2015). It has been widely used in the fields such as information systems outsourcing (Chen, Wang, \& Wu, 2011b), energy exploitation (Goumas \& Lygerou, 2000), selection of equipment (Yilmaz \& Dağdeviren, 2011) and material (Gul, Celik, Gumus, \& Guneri, 2017), health care management (Amaral \& Costa, 2014) and ERP (Kilic, Zaim, \& Delen, 2015), selection of supplier (Krishankumar, Ravichandran, \& Saeid, 2017) or countries for developmental aid (Afful-Dadzie, Nabareseh, Oplatková, \& Klimek, 2016) as well. Subsequently, the fuzzy AHP and the PROMETHEE were introduced in the areas such as the selection of additive manufacturing process (Peko et al., 2018), evaluation of solar power plant location alternatives (Samanlioglu \& Ayağ, 2017), disassembly line balancing problem (Avikal, Mishra, \& Jain, 2014), power substation location selection (Kabir \& Sumi, 2014), etc.

In order to take the qualitative evaluation information into account, linguistic PROMETHEE was applied to handle the decision-making problem of investment portfolio as well (Chen, et al. 2011a). It was used to deal with the problem such as the assessment of quality of portal website service (Lee \& Chang, 2010), energy planning (Singh, Gupta, \& Mehra, 2016), third-party logistics supplier selection (Chen, Pai, \& Hung, 2010), and so on. Considering that the decision-making is the result of swarm intelligence, Halouani, Chabchoub, and Martel (2009) extended the PROMETHEE to group decision making and developed a PROMETHEE-MD-2T method to select a project.

From the literature reviews above, it is known that the PROMETHEE has been extended in several fuzzy circumstances and applied in various fields. Although Afful-Dadzie et al. (2015) discussed the fuzzy PROMETHEE to select start-up firm for public VC, it is a decision model referring to triangular fuzzy information. While in this paper we concentrate on hesitant fuzzy linguistic information which is generally used in the VCs' evaluation process. Furthermore, the group decision-making problem ignored by Afful-Dadzie et al. (2015) is considered in this paper, which is in accordance with the reality. In addition, there is no research focused on combining the PT with the PROMETHEE under hesitant fuzzy linguistic circumstance and on its application in aiding the VCs to select a promising start-up firm simultaneously. Therefore, in this paper, we attempt to construct such a comprehensive method to identify a promising start-up firm. 


\section{Preliminaries}

Because the key point of this paper is to explore the bounded rationality of VCs, the PT is reviewed firstly in this section. Then, due to the fact that the linguistic information is generally used by VCs, some basic concepts and algorithms are presented as well.

\subsection{Prospect theory}

PT is a great innovation to describe behavioral decision-making of individuals under bounded rationality through the prospect value $V\left(x_{i}\right)$ which is the product of the value function $v\left(x_{i j}\right)$ and the probability weighting function $w\left(p_{j}\right)$.

$$
\begin{aligned}
& V\left(x_{i}\right)=\sum_{j=1}^{m} v\left(x_{i j}\right) w\left(p_{j}\right) \\
& v\left(x_{i j}\right)=\left\{\begin{array}{ll}
-\lambda\left(x_{0}-x_{i j}\right)^{\beta} & x_{i j}-x_{0}<0 \\
\left(x_{i j}-x_{0}\right)^{\alpha} & x_{i j}-x_{0} \geq 0
\end{array} ;\right. \\
& w\left(p_{j}\right)=\left\{\begin{array}{ll}
\frac{p_{j}^{\delta}}{\left[p_{j}^{\delta}+\left(1-p_{j}\right)^{\delta}\right]^{1 / \delta}} & x_{i j}-x_{0}<0 \\
\frac{p_{j}^{\gamma}}{\left[p_{j}^{\gamma}+\left(1-p_{j}\right)^{\gamma}\right]^{1 / \gamma}} & x_{i j}-x_{0} \geq 0
\end{array},\right.
\end{aligned}
$$

where $x_{i j}$ is the value of the ith alternative over the criterion $c_{j}$; $x_{0}$ is the reference point; $p_{j}$ is the probability/weighting value of the criterion $c_{j} ; \beta, \lambda, \alpha, \delta$ and $\gamma$ are the parameters obtained from experimental economics. The parameters used in Section 4 came from the classical experiment (Tversky \& Kahneman, 1992), which have been accepted by the majority of scholars. Hence, in this paper $\alpha=\beta=0.88, \lambda=2.25, \delta=0.69$ and $\gamma=0.61$. Additionally, PT has been used to deal with the problems such as emergency decision-making methods (L. Wang, Y. M. Wang, \& Martínez, 2018; L. Wang, Labella, Rodríguez, Y. M. Wang, \& Martínez, 2017). Seldom of the existing research considers the psychological behavior of VCs in the decision-making process.

\subsection{Hesitant fuzzy linguistic information}

The hesitant fuzzy linguistic information is expressed by the HFLTSs which were proposed by Rodríguez et al. (2012) to deal with the qualitative MCDM problems under uncertain situations in which the VCs are hesitant between several linguistic terms to evaluate business projects. For instance, when the VCs are asked to evaluate the product or the service of a start-up firm, they are more likely to adopt the word such as "at least good", "medium", "a little bad", and so on. In such cases, it is easy for us to transform those linguistic expressions into the HFLTSs (please see the example in the last paragraph of Section 4.1). Since we concentrate on constructing group decision-making models, we just need to understand this linguistic transformation (please refer to Rodríguez et al. $(2012,2013)$ Rodríguez, Álvaro, and Martínez (2016) for more details). 
Since the linguistic variable was proposed by Zadeh (1975a, 1975b, 1975c), it has been extended in various forms. Other techniques to model complex linguistic expressions such as linguistic 2-tuple (Herrera \& Martínez, 2000), virtual linguistic (Xu, 2005), hesitant fuzzy linguistic (Rodríguez et al., 2012), etc. have been developed. Let $S=\left\{s_{t} \mid t=-\varsigma, \cdots,-1,0,1, \cdots, \varsigma\right\}$ be a subscript-symmetric linguistic term set $(\mathrm{Xu}, 2005)$. Then, the HFLTS can be defined as $H_{S}(x)=<x, s_{t_{l}}(x) \in S \mid x \in X>\left(l=1, \cdots, \# H_{s}\right)$ (Liao, Xu, \& Zeng, 2014), where $x$ is an alternative while $X$ is the set of alternatives, and $\# H_{s}$ is the number of linguistic terms. It is necessary for us to introduce the comparison rules for any two HFLTSs (Liao, Xu, \& Zeng, 2015; Wei, Rodríguez, \& Martínez, 2018), which is important to the practical application. At first, the score function and the variance function of $H_{s}$ should be presented (Liao et al., 2015).

$$
\begin{gathered}
\rho\left(H_{S}\right)=\frac{1}{\# H_{S}} \sum_{s_{t_{l}} \in H_{S}} s_{t_{l}}=s_{\frac{1}{\# H_{S}} \sum_{l=1}^{\# H_{S}}} ; \\
\sigma\left(H_{S}\right)=\frac{1}{\# H_{S}} \sqrt{\sum_{s_{t_{l}}, s_{t_{g}} \in H_{S}}\left(s_{t_{l}}-s_{t_{g}}\right)^{2}}=s_{\frac{1}{\# H_{S}} \sqrt{\sum_{s_{t_{l}}, s_{t_{g}} \in H_{S}}\left(\mathrm{t}_{l}-t_{g}\right)^{2}}}
\end{gathered}
$$

Next, the comparison rules (Liao et al., 2015) for two HFLTSs $H_{s}$ and $H_{s}{ }^{\prime}$ will be:

(1) If $\rho\left(H_{S}\right)>\rho\left(H_{S}{ }^{\prime}\right)$, then $H_{S} \succ H_{S}{ }^{\prime}$;

(2) If $\rho\left(H_{S}\right)<\rho\left(H_{S}{ }^{\prime}\right)$, then $H_{S} \prec H_{S}{ }^{\prime}$;

(3) If $\rho\left(H_{S}\right)=\rho\left(H_{S}{ }^{\prime}\right)$, in this situation, the variance function can be introduced for further comparison: a) If $\sigma\left(H_{S}\right)>\sigma\left(H_{S}{ }^{\prime}\right)$, then $H_{S} \prec H_{S}{ }^{\prime}$; b) If $\sigma\left(H_{S}\right)<\sigma\left(H_{S}{ }^{\prime}\right)$, then $H_{S} \succ H_{S}^{\prime}$; c) If $\sigma\left(H_{S}\right)=\sigma\left(H_{S}^{\prime}\right)$, then $H_{S} \approx H_{S}{ }^{\prime}$.

Furthermore, \# $H_{s}=\# H_{s}{ }^{\prime}$ is the precondition of the distance measure of any two HFLTSs. If there is $\# H_{s} \neq \# H_{s}{ }^{\prime}$, then we should add linguistic terms for the shorter one. For instance, if there is $\# H_{s}<\# H_{s}{ }^{\prime}$, then we let $H_{s}^{+}=\max \left\{s_{l} \mid s_{l} \in H_{s}\right\}$ and $H_{s}^{-}=\min \left\{s_{l} \mid s_{l} \in H_{s}\right\}$ be the maximum and minimum linguistic terms in $S$ respectively. Then, according to Wei, Ren, and Rodríguez (2015), the added linguistic term is:

$$
\hat{s}=C^{2}\left(\xi, H_{s}^{+}, 1-\xi, H_{s}^{-}\right)=\xi \odot H_{s}^{+} \oplus(1-\xi) \odot H_{s}^{-},
$$

where $\xi(0 \leq \xi \leq 1)$ is the parameter that reflects the risk preferences of the decision makers and $C^{2}\left(\xi, H_{s}^{+}, 1-\xi, H_{s}^{-}\right)$is the convex combination of two linguistic terms (Delgado, Verdegay, \& Vila, 1993). In this paper, it is assumed that $\xi=1$. In other words, we should add the max linguistic term to the shorter one. For instance, if there are two HFLTSs $H_{1}=\left\{s_{0}, s_{1}, s_{2}, s_{3}\right\}$ and $H_{2}=\left\{s_{0}, s_{1}, s_{2}\right\}$, then, according to Eq. (6), we should add the linguistic term $s_{2}$ to $H_{2}$, and get the extended $H_{2}=\left\{s_{0}, s_{1}, s_{2}, s_{2}\right\}$. After that, the distance measure between $H_{1}$ and $H_{2}$ is exhibited. There are many kinds of distance measures for the HFLTSs such as the Hamming distance, the Euclidean distance, the Hausdorff distance and their extensions. Since it is not the main focus in this paper, the Hamming distance for the HFLTSs is adopted:

$$
d\left(H_{S}, H_{S}{ }^{\prime}\right)=\frac{1}{\# H_{S}} \sum_{l=1}^{\# H_{S}} \frac{\left|t_{l}-t_{l}^{\prime}\right|}{2 \varsigma+1} .
$$

Generally, there are two kinds of attributes in the decision-making processes: cost attributes and benefit attributes. The smaller the value of the cost attribute is, the better it will be. 
On the contrary, the bigger the value of the benefit attribute is, the better it will be. Therefore, for the sake of convenience, we should unify those different attributes. In this paper, we transform the cost attributes to the benefit ones. For the benefit attribute, ${ }_{b} H_{S}={ }_{b} H_{S}$. While for the cost attribute, ${ }_{c} H_{S}=\overline{{ }_{c} H_{S}}$, where ${ }_{c} H_{S}$ is the inverse operation of the HFLTS with $\overline{{ }_{c} H_{S}}=\left\{s_{-i} \mid s_{i} \in{ }_{c} H_{S}\right\}$.

From the above analysis, it is sufficient for us to integrate the PT into the PROMETHEE under the hesitant fuzzy linguistic circumstance for the VCs to make a more reasonable decision.

\section{Construction of group decision-making methods with the PROMETHEE under hesitant fuzzy linguistic circumstance}

In this section, two group decision-making models are constructed according to the analysis above. Also, the detailed steps of them are given, including a visual procedure for understanding the decision-making process easily.

\subsection{G-PROMETHEE with hesitant fuzzy linguistic information}

The PROMETHEE is a family of methods developed to solve different kinds of ranking problems. For example, the PROMETHEEs I and II were introduced for partial and complete rankings of alternatives correspondingly (Brans, 1982). The PROMETHEE III is a ranking method on the basis of intervals. Later, it was extended to the continuous case that is called PROMETHEE IV (Brans et al., 1986). The PROMETHEE V was given by Brans and Mareschal (1992), which is efficient in the problem with segmentation constraints. The PROMETHEE VI was built to represent the human brain (Brans \& Mareschal, 1995). Some extensions like the GDSS PROMETHEE (Macharis, Brans, \& Mareschal, 1998), the IF-PROMETHEE (Liao \& Xu, 2014), etc., have been established. Although the PROMETHEE has been expanded to various forms, the main idea of the conventional PROMETHEE, which is to select the promising alternative according to the net flow, still exists in those extensions.

The conventional PROMETHEE concentrates on the pairwise comparison for each criterion with crisp numbers, which could not simulate the qualitative information well. Moreover, as the deepening of the social division of labor and the specialization of individuals, group decision making has been a process of solving the decision-making problems in most cases. Treville, Petty, and Wager (2014) investigated that VCs only interested $20 \%$ of proposals among the 3631 proposals during 11 years. But due to the lack of capacity, $9 \%$ of those interesting deals were not accessed by the VCs, and again they lost some opportunities. Thus, group decision making which takes the advantage of each group member can avoid such situations and recognizes opportunities easily.

Taking the above phenomena into consideration, it is of importance to establish a GPROMETHEE under hesitant fuzzy linguistic circumstance. Following the steps below, the ranking results will be obtained based on the G-PROMETHEE with hesitant fuzzy linguistic information.

Step 1. Acquire the original evaluation information under hesitant fuzzy linguistic circumstance. 


$$
X^{k}=\left(\begin{array}{ccc}
x_{11}^{k} & \cdots & x_{1 m}^{k} \\
\vdots & \ddots & \vdots \\
x_{n 1}^{k} & \cdots & x_{n m}^{k}
\end{array}\right),
$$

where $n$ is the number of start-up firms; $m$ is the number of criteria; $x_{i j}^{k}$ is the $k$ th VCs' evaluation information for the start-up firm $x_{i}$ over the criterion $c_{j}$. For convenience, let $N=\{1,2, \cdots, n\}, M=\{1,2, \cdots, m\}$ and $Z=\{1,2, \cdots, z\}$. Then, $i \in N, j \in M$ and $k \in Z$.

Step 2. Translate the original evaluation information into the hesitant fuzzy linguistic judgment matrix, and then convert the cost attributes into the benefit ones according to the content in Section 2.2.

$$
\mathbf{H}^{k}=\left(\begin{array}{ccc}
H_{11}^{k} & \cdots & H_{1 m}^{k} \\
\vdots & \ddots & \vdots \\
H_{n 1}^{k} & \cdots & H_{n m}^{k}
\end{array}\right)
$$

where $H_{i j}^{k}=\left(x_{i j}^{k}, h_{S}\left(x_{i j}^{k}\right) \mid x_{i j}^{k} \in X\right)=\left\{<x_{i j}^{k},\left[s_{t_{l}}\left(x_{i j}^{k}\right) \mid s_{t_{l}}\left(x_{i j}^{k}\right) \in S, l=1,2, \cdots, \# H_{i j}^{k}\right]>\mid x_{i j}^{k} \in X\right\}$, which denotes the possible evaluation values from the $k$ th VCs for the $i$ th start-up firm over the $j$ th criterion, that is, the $k$ th VCs expresses his/her evaluation information on $x_{i j}$ with $H_{i j}^{k}$; Moreover, $H_{i j}^{k}$ is the standardized evaluation information according to the laws in Section 2.2. For instance, $H_{i j}^{k}=H_{i j_{b}}^{k}$ when $j_{b}$ is the benefit criterion, whereas, $H_{i j}^{k}=H_{i j_{c}}^{k}$ when $j_{c}$ is the cost criterion.

Step 3. Identify the deviation between the start-up firms on the basis of Eq. (10):

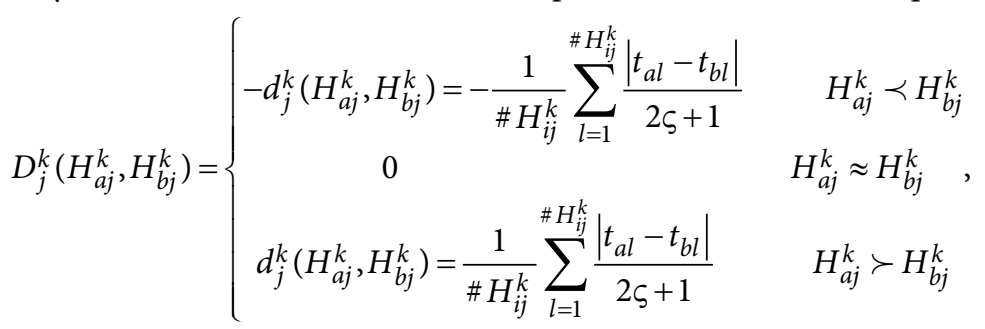

where $\forall a \in N, \forall b \in N, i \in N$ and $j \in M$. Moreover, $d_{j}^{k}\left(H_{a j}^{k}, H_{b j}^{k}\right)$ is the distance between $H_{a j}$ and $H_{b j}$. When there is $H_{a j}^{k} \prec H_{b j}^{k}$, which is acquired from the comparison rules in Section 2.2, the deviation between them is the opposite number of the distance. In contrast, when there is $H_{a j}^{k} \succ H_{b j}^{k}$, the deviation between them is the distance. Actually, the deviation between $H_{a j}$ and $H_{b j}$ is a relative concept. It means that, relative to $H_{b j}$, the deviation of $H_{a j}$ and $H_{b j}$ is negative when $H_{a j}^{k} \prec H_{b j}^{k}$ and is positive when $H_{a j}^{k} \succ H_{b j}^{k}$.

Step 4. Determine the preference according to the preference function:

$$
P_{j}^{k}\left(x_{a}^{k}, x_{b}^{k}\right)=F_{j}^{k}\left(D_{j}^{k}\right),
$$

where $D_{j}^{k}\left(H_{a j}^{k}, H_{b j}^{k}\right)$ is the independent variable of the preference function $F(\cdot)$. There have been six types of preference functions (Brans et al., 1986). The type V with linear preference and indifference area has been chosen as the representation of preference function of the VCs in this paper: 


$$
F_{j}\left(D_{j}^{k}\right)=\left\{\begin{array}{cl}
-1 & \text { if } D_{j}^{k} \leq-\bar{q}_{j} \\
-\frac{D_{j}^{k}+\underline{q}_{j}}{\underline{q}_{j}-\bar{q}_{j}} & \text { if }-\bar{q}_{j}<D_{j}^{k} \leq-\underline{q}_{j} \\
0 & \text { if }-\underline{q}_{j}<D_{j}^{k} \leq \underline{q}_{j}, \\
\frac{D_{j}^{k}-\underline{q}_{j}}{\bar{q}_{j}-\underline{q}_{j}} & \text { if } \underline{q}_{j}<D_{j}^{k} \leq \bar{q}_{j} \\
1 & \text { if } \bar{q}_{j}<D_{j}^{k}
\end{array}\right.
$$

where $\underline{q}_{j}$ is the indifference threshold and $\bar{q}_{j}$ is the strict preference threshold.

Step 5. Aggregate the global preference index:

$$
G^{k}\left(x_{a}^{k}, x_{b}^{k}\right)=\sum_{j=1}^{m} \omega_{j} P_{j}^{k}\left(x_{a}^{k}, x_{b}^{k}\right),
$$

where $\omega_{j}$ is the weight of the $j$ th attribute.

Step 6. Calculate the positive outranking flow and the negative outranking flow of the VCs correspondingly:

$$
\begin{aligned}
\phi^{k+}\left(x_{a}^{k}\right) & =\frac{1}{n-1} \sum_{i \in N} G^{k}\left(x_{a}^{k}, x_{i}^{k}\right) ; \\
\phi^{k-}\left(x_{a}^{k}\right) & =\frac{1}{n-1} \sum_{i \in N} G^{k}\left(x_{i}^{k}, x_{a}^{k}\right) .
\end{aligned}
$$

Step 7. Get the net outranking flow of the VCs:

$$
\phi^{k}\left(x_{a}^{k}\right)=\phi^{k+}\left(x_{a}^{k}\right)-\phi^{k-}\left(x_{a}^{k}\right) .
$$

Step 8. Integrate the net outranking flow of each venture capitalist:

$$
\psi\left(x_{a}\right)=\sum_{k=1}^{z} \pi_{k} \phi^{k}\left(x_{a}^{k}\right),
$$

where $\pi_{k}$ is the $k$ th venture capitalist's weight and it is calculated by the entropy method. As we all know that the entropy is used to measure the degree of disorder in a system. The higher disorder of the system is, the larger of the entropy value will be. While, for a group decision-making problem, if a venture capitalist shows a similar assessment for each start-up firm, then the entropy value of this venture capitalist will be larger, and his/her weight will be smaller. Thus,

$$
\pi_{k}=\frac{1-e_{k}}{z-\sum_{k=1}^{z} e_{k}}
$$

where $e_{k}$ is the entropy value of the $k$ th venture capitalist, and it can be obtained from Eq. (19) and Eq. (20):

$$
e_{k}=-\frac{1}{\ln n} \sum_{i=1}^{n}\left(\frac{\varphi_{i}^{k}}{\sum_{i=1}^{n} \varphi_{i}^{k}} * \ln \left|\frac{\varphi_{i}^{k}}{\sum_{i=1}^{n} \varphi_{i}^{k}}\right|\right)
$$




$$
\varphi_{i}^{k}=\left(\sum_{j=1}^{m} \frac{\sum_{l=1}^{\# H_{i j}^{k}} t_{l}}{\# H_{i j}^{k}}\right) / m .
$$

The promising start-up firm will be found out according to the ranking order of $\psi\left(x_{a}\right)$. The bigger $\psi\left(x_{a}\right)$ is, the better the start-up firm $x_{a}$ will be. If there are equal $\psi\left(x_{a}\right)$ and $\psi\left(x_{a^{\prime}}\right)$, then we should turn to Step 9. Otherwise, we should go to Step 10.

Step 9. If $\psi\left(x_{a}\right)=\psi\left(x_{a^{\prime}}\right)$, then the Eq. (22) should take the place of the Eq. (20) in Eq. (19). In this situation, the entropy weight of the $k$ th venture capitalist will be obtained by using Eq. (21) and Eq. (22):

$$
\begin{gathered}
e_{k}=-\frac{1}{\ln n} \sum_{i=1}^{n}\left(\frac{\varphi_{i}^{\prime k}}{\sum_{i=1}^{n} \varphi_{i}^{\prime k}} * \ln \left|\frac{\varphi_{i}^{\prime k}}{\sum_{i=1}^{n} \varphi_{i}^{\prime k}}\right|\right) ; \\
\varphi_{i}^{\prime k}=\left(\sum_{j=1}^{m} \frac{\sqrt{\sum_{s_{t_{l}, s_{t} \in H_{i j}^{k}}\left(\mathrm{t}_{l}-t_{g}\right)^{2}}^{\#}}}{\# H_{i j}^{k}}\right) / m .
\end{gathered}
$$

Step 10. End.

The detailed steps of G-PROMETHEE with hesitant fuzzy linguistic information has been established, but it could not simulate the psychological state of VCs. Thus, in the next section, a more generalized model that considers the effect of psychological factors of VCs on their decision-making is constructed.

\subsection{GP-PROMETHEE with hesitant fuzzy linguistic information}

As the literature shown in Section 1.1, the fuzzy information has been infused in the PROMETHEE to depict uncertainty, but the uncertainty comes not only from the objective affairs but also from the bounded perceptions of the VCs for objective affairs. Thus, it is necessary to take into consideration the role of the perceived values derived from the VCs in the decisionmaking process. Although the intuitionistic fuzzy PROMETHEE (Liao \& Xu, 2014) considers both the intuitionistic fuzzy preference and the intuitionistic fuzzy weight, it ignores the effect of psychological state of the decision makers on the final decision. The decision makers are individuals who are quite different in gender, experience, education, and so on, which leads to different risk preferences. Therefore, in consideration of risk preferences of the decision makers, a generalized PROMETHEE is built (Shih, Chang, \& Cheng, 2016). Although the risk preferences on gains and losses are depicted via modifying the preference function with the parameters of the PT, the transformed probability is not considered in this generalized model. Another research takes the deviation of the value function of the PT instead of the original evaluation values as the independent variable of the preference function in the PROMETHEE (Lerche \& Geldermann, 2015). Both of those two researches attempted to 
integrate the PT into the PROMETHEE and the different risk attitudes on gains and losses have been considered indeed, but none of them take into account the subjective probability which is the important part of the PT. Moreover, the evaluation information was expressed as crisp numbers in those two researches, which is hard for the decision makers to express their opinions accurately. Additionally, the group decision making should be considered as well.

Although the group decision-making method established in Section 3.1 includes the hesitant fuzzy linguistic situation of the VCs, it could not simulate the risk attitudes of them. The attitude such as risk aversion for gains or risk seeking for losses is the obvious phenomena among the VCs. Taking those psychological factors of the VCs into account is a necessary way to make a better decision. It is the aim of this section to construct a group decisionmaking method which integrates the merit of both the PT and the hesitant fuzzy linguistic information named GP-PROMETHEE to well portray the psychological factors and the evaluation information of the VCs. The detailed steps can be given as follows:

Step 1. Obtain the original evaluation information from the VCs under hesitant fuzzy linguistic circumstance.

Step 2. Transform the evaluation matrix into the HFLTSs and convert the cost attributes into the benefit ones.

Step 3. Calculate the deviation of prospect value between the start-up firms $x_{a}$ and $x_{b}$ over the criterion $c_{j}$ based on the hesitant fuzzy linguistic matrix:

$$
\begin{gathered}
V_{j}^{k}\left(x_{a}^{k}, x_{b}^{k}\right)=v_{j}\left(x_{a}^{k}, x_{b}^{k}\right) w\left(p_{j}\right) ; \\
v_{j}\left(x_{a}^{k}, x_{b}^{k}\right)=\left\{\begin{array}{cc}
-\lambda\left(d_{j}\left(H_{a j}^{k}, H_{b j}^{k}\right)\right)^{\beta} & H_{a j}^{k} \prec H_{b j}^{k} \\
0 & H_{a j}^{k} \approx H_{b j}^{k} \\
\left(d_{j}\left(H_{a j}^{k}, H_{b j}^{k}\right)\right)^{\alpha} & H_{a j}^{k} \succ H_{b j}^{k}
\end{array}\right. \\
w\left(p_{j}\right)= \begin{cases}\frac{p_{j}^{\delta}}{\left[p_{j}^{\delta}+\left(1-p_{j}\right)^{\delta}\right]^{1 / \delta}} & H_{a j}^{k} \prec H_{b j}^{k} \\
\frac{p_{j}^{\gamma}}{\left[p_{j}^{\gamma}+\left(1-p_{j}\right)^{\gamma}\right]^{1 / \gamma}} & \text { otherwise }\end{cases}
\end{gathered}
$$

where $\forall a \in N, \forall b \in N, \forall j \in M$ and $\forall k \in Z$; the relationship between $H_{a j}^{k}$ and $H_{b j}^{k}$ is determined by the comparison rules in Section 2.2; $d\left(H_{a j}^{k}, H_{b j}^{k}\right)$ is the distance between the start-up firms $x_{a}$ and $x_{b}$ over the criterion $c_{j}$, and it is acquired from Eq. (7); $p_{j}$ is the importance degree of $c_{j}$ given by the VCs, in other words, it is the original weight of the attribute $c_{j}$.

Step 4. Work out the VCs' preferences according to the preference function:

$$
P_{j}^{k}\left(x_{a}^{k}, x_{b}^{k}\right)=F_{j}\left(V_{j}^{k}\right),
$$

where $V_{j}^{k}$ is acquired from Eq. (23) and it is the independent variable of the preference function $F(\cdot)$. Here $F(\cdot)$ adopts Eq. (12). 
Step 5. Aggregate the global preference index of the VCs:

$$
G^{k}\left(x_{a}^{k}, x_{b}^{k}\right)=\sum_{j=1}^{m} P_{j}^{k}\left(x_{a}^{k}, x_{b}^{k}\right) .
$$

Then, following Steps 6 to 10 in Section 3.1, the ranking results will be acquired and also the promising start-up firm will be recognized.

Until now, the detailed steps of the GP-PROMETHEE have been constructed. To be understood easily, a visual procedure has been drawn up as well (see Figure 1).

Since the detailed steps and visual procedure of selecting the promising start-up firm have been finished, an illustrative example will be presented in Section 4 to verify the reasonability and feasibility of the GP-PROMETHEE.

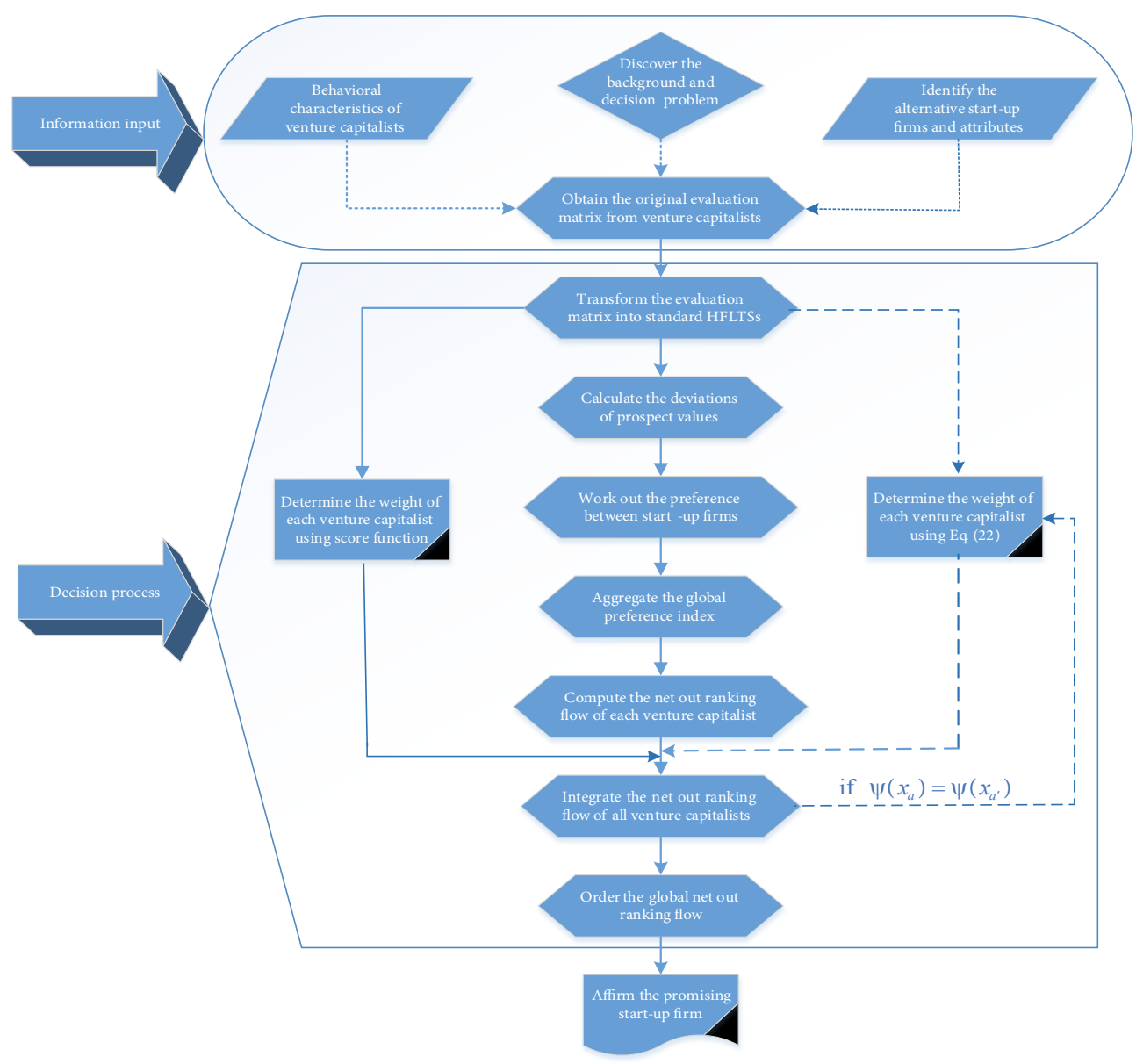

Figure 1. The visual procedure of the GP-PROMETHEE for the VCs 


\section{Illustrative example for selecting the promising start-up firms}

After constructing the detailed steps of the group decision-making models, the feasibility and effectiveness of the proposed models are presented in this section through an example. Meanwhile, a TODIM, which is an outranking method based on PT, is used in the same example for the sake of showing the stability of the GP-PROMETHEE. Then, the comparative analyses are presented here.

\subsection{Decision-making background of the VCs}

In this section, an example from the Sinovation Ventures is provided. Numerous VCs in Sinovation Venture are preoccupied by selecting the promising start-up firm to gain extra revenue and bear high risk at the same time. Owing to the merge of DiDi and Uber and also the intervention of the government, the form of online ordering car has been out of the public eye gradually. Since the year of 2015, the bike-share program has received much attentions from all the society including the VCs in China. It is a new structure of business which satisfies individuals' vehicle need for short distance with lower cost and convenient cycling instead of walk. The idea of 'Green travel, enjoy cycling' has been accepted by more and more individuals. Furthermore, the government actively advocates citizens to choose a healthy and lower carbon way to travel which is beneficial not only to decrease the emission of carbon dioxide but also to relieve the urban traffic jam problems. Hence, as the most popular business program at present, the promising company with bike-share program is worthy of pursuing by the VCs.

There are several competitive start-up firms in the bike-share market such as 'ofo', 'Xiaoming bike', 'mobike', 'U-Bicycle', 'Panda bike', etc. After preliminary investigation by the interviewees, four competitive bike-share companies remain to be comprehensive evaluation, named 'ofo', 'Xiaoming bike', 'mobike' and 'U-Bicycle'. Moreover, $x_{1}, x_{2}, x_{3}$ and $x_{4}$ have been used to represent those alternative bike-share companies correspondingly. After furious discussion in the evaluation process, our interviewees have reached the consistent opinion. They have agreed to make their decisions according to the following criteria: (1) $c_{1}$, the management team of the bike-share company (such as the educational background of the team member, the experience of the team member, the effort level of the team member, etc.); (2) $c_{2}$, the market potential of the bike-share program; (3) $c_{3}$, the service quality of the bike-share company (such as customer satisfaction, the market acceptance, etc.); (4) $c_{4}$, the financial situation of the bike-share company (including the potential revenue, the asset liquidity, the uncertain investment cost, etc.). In this paper, those four criteria are adopted as the gist of decision-making for the VCs. As has been mentioned above, they are hard to be quantified as crisp numbers. Therefore, using the hesitant fuzzy linguistic information to portray the perceptions of the VCs is suitable.

Although the criteria are correlated with different contexts so that different criteria will be depicted with different linguistic term sets. Fortunately, the contexts of those four criteria associated in this VC environment are the same. Thus, the linguistic term sets of the four criteria are shown as: 
$S_{C_{j}}=\left\{s_{-3}=\right.$ very bad, $s_{-2}=$ bad, $s_{-1}=$ a little bad, $s_{0}=$ medium, $s_{1}=$ a little good, $s_{2}=$ good, $s_{3}=$ very good $\}$,

where $c_{j}$ is the $j$ th criterion/attribute. The VCs will express their evaluation information with hesitant fuzzy linguistic forms. For example, the $k$ th venture capitalist thinks that the management team of 'mobike' is 'at least good'. Next, the evaluation information will be translated to the HFLTSs: $H_{11}^{k}=\left(x_{11}^{k}, h_{S}\left(x_{11}^{k}\right)\right)=\left\{<x_{11}^{k},\left[s_{2}, s_{3}\right]>\right\}$, because 'at least good' means 'good' or 'very good' which is represented by $s_{2}$ or $s_{3}$ correspondingly (please refer to Rodríguez et al. (2012) for more detailed explanations). Considering the limited capacity of each person and the advantage of group decision making, three senior investors served as our interviewees have been invited to evaluate the remaining bike-share firms $\left(x_{1}, x_{2}, x_{3}, x_{4}\right)$ from four criteria $\left(c_{1}, c_{2}, c_{3}, c_{4}\right)$. By adopting the criteria of six countries/regions discussed by (Widyanto \& Dalimunthe, 2015) and the suggestions of our interviewees, the criteria weights are: $\omega_{1}=0.535=p_{1}, \omega_{2}=0.086=p_{2}, \omega_{3}=0.23=p_{3}$ and $\omega_{4}=0.149=p_{4}$. Also, the indifference threshold is supposed to be zero under all attributes. It means that any tiny discrepancies between bike-share firms will produce relative preferences. The strict preference threshold expresses that if the independent variable is greater than the strict preference threshold, the preference degrees of the VCs to a particular bike-share firm will be the values of the preference function. That is to say, the smaller the strict preference threshold for a specific attribute is, the more sensitivity the VCs for this attribute will be of. According to the attributes that the VCs care about, the strict preference thresholds of those attributes are supposed to be $\bar{q}_{1}=0.2, \bar{q}_{2}=0.3, \bar{q}_{3}=0.25$ and $\bar{q}_{4}=0.6$. Afterwards, following the steps, the promising bike-share company will be found.

\subsection{Results of the G-PROMETHEE with hesitant fuzzy linguistic information}

Step 1. The original evaluation information (linguistic expressions) from the VCs under hesitant fuzzy linguistic circumstance will be collected. The VCs know that their linguistic expressions conform to the linguistic term sets $S_{C_{j}}$ mentioned in Section 4.1. Taking the venture capitalist 1 as an example, his/her evaluation information is shown in Table 1.

Table 1 . The original evaluation information from the venture capitalist 1

\begin{tabular}{|c|c|c|c|c|}
\hline Start-up firms & $c_{1}$ & $c_{2}$ & $c_{3}$ & $c_{4}$ \\
\hline$x_{1}$ & $\begin{array}{c}\text { Bettributeen a little } \\
\text { good and good }\end{array}$ & $\begin{array}{c}\text { Between medium } \\
\text { and good }\end{array}$ & $\begin{array}{c}\text { Between medium } \\
\text { and a little good }\end{array}$ & $\begin{array}{c}\text { Between medium } \\
\text { and a little good }\end{array}$ \\
\hline$x_{2}$ & $\begin{array}{c}\text { Between medium } \\
\text { and a little good }\end{array}$ & $\begin{array}{c}\text { Between bad and } \\
\text { a little bad }\end{array}$ & $\begin{array}{c}\text { Between a little } \\
\text { bad and a little } \\
\text { good }\end{array}$ & $\begin{array}{c}\text { Between a little } \\
\text { bad and medium }\end{array}$ \\
\hline$x_{3}$ & At least good & $\begin{array}{c}\text { Between medium } \\
\text { and good }\end{array}$ & A little good & $\begin{array}{c}\text { Between a little } \\
\text { good and good }\end{array}$ \\
\hline$x_{4}$ & Between medium & $\begin{array}{c}\text { Between a little } \\
\text { and a little good and medium }\end{array}$ & $\begin{array}{c}\text { Between a little } \\
\text { bad and medium }\end{array}$ & $\begin{array}{c}\text { Between a little } \\
\text { bad and a little } \\
\text { good }\end{array}$ \\
\hline
\end{tabular}


Step 2. As the example stated in last paragraph of Section 4.1, the linguistic expressions are represented by the corresponding linguistic terms in $S_{C_{j}}$. According to the transformation function $E$ (Rodríguez et al., 2012), the linguistic expressions in Table 1 are transformed into HFLTSs. The transformed rules of $E$ will be:

(1) $E\left(s_{t}\right)=\left\{s_{t} / s_{t} \in S\right\}$;

(2) E(less than $\left.s_{t}\right)=\left\{s_{t^{\prime}} / s_{t^{\prime}} \in S\right.$ and $\left.s_{t^{\prime}} \leq s_{t}\right\}$;

(3) E(at least $\left.s_{t}\right)=\left\{s_{t^{\prime}} \mid s_{t^{\prime}} \in S\right.$ and $\left.s_{t^{\prime}} \geq s_{t}\right\}$;

(4) E(between $s_{t}$ and $\left.s_{t^{\prime}}\right)=\left\{s_{t^{\prime \prime}} \mid s_{t^{\prime \prime}} \in S\right.$ and $\left.s_{t} \leq s_{t^{\prime \prime}} \leq s_{t^{\prime}}\right\}$.

For instance, for the linguistic expression "Between medium and good", there is a transformation function $E$ (between medium and good $)=\{$ medium, a little good, good $\}$, then, the corresponding HFLTS is $H=\left\{s_{0}, s_{1}, s_{2}\right\}$ (more details about transformation function please refer to Rodríguez et al., 2012). Thereby, the original evaluation information (linguistic expressions) provided by all the VCs such as in Table 1 are transformed into the standardized hesitant fuzzy linguistic evaluation matrices as $\mathbf{H}^{1}, \mathbf{H}^{2}$ and $\mathbf{H}^{3}$ respectively:

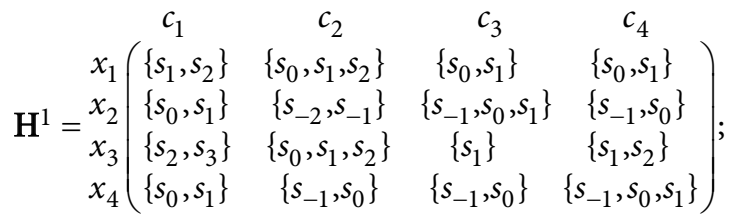

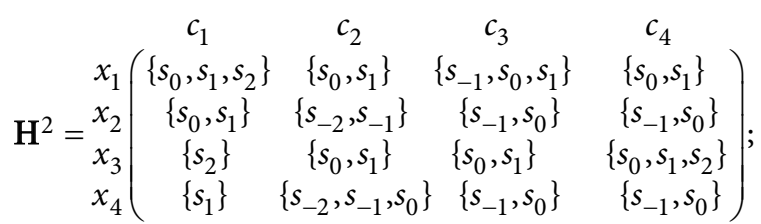

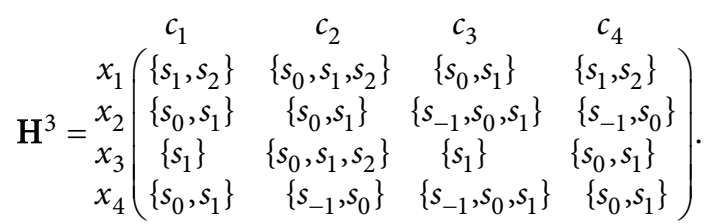

Step 3. The differences between the start-up firms are shown in Table 2.

Table 2. Deviations of the start-up firms from VCs

\begin{tabular}{|c|c|c|c|c|c|c|c|c|c|c|c|c|}
\hline Attributes & \multicolumn{4}{|c|}{ Venture capitalist 1} & \multicolumn{4}{c|}{ Venture capitalist 2} & \multicolumn{4}{c|}{ Venture capitalist 3} \\
\cline { 2 - 15 } & $c_{1}$ & $c_{2}$ & $c_{3}$ & $c_{4}$ & $c_{1}$ & $c_{2}$ & $c_{3}$ & $c_{4}$ & $c_{1}$ & $c_{2}$ & $c_{3}$ & $c_{4}$ \\
\hline$D_{j}^{k}\left(H_{1 j}^{k}, H_{2 j}^{k}\right)$ & 0.143 & 0.333 & 0.095 & 0.143 & 0.048 & 0.286 & 0.048 & 0.143 & 0.143 & 0.048 & 0.095 & 0.286 \\
\hline$D_{j}^{k}\left(H_{1 j}^{k}, H_{3 j}^{k}\right)$ & -0.143 & 0 & -0.071 & -0.143 & -0.143 & 0 & -0.095 & -0.048 & 0.071 & 0 & -0.071 & 0.143 \\
\hline$D_{j}^{k}\left(H_{1 j}^{k}, H_{4 j}^{k}\right)$ & 0.143 & 0.190 & 0.143 & 0.095 & -0.095 & 0.238 & 0.048 & 0.143 & 0.143 & 0.190 & 0.095 & 0.143 \\
\hline$D_{j}^{k}\left(H_{2 j}^{k}, H_{1 j}^{k}\right)$ & -0.143 & -0.333 & -0.095 & -0.143 & -0.048 & -0.286 & -0.048 & -0.143 & -0.143 & -0.048 & -0.095 & -0.286 \\
\hline
\end{tabular}


End of Table 2

\begin{tabular}{|c|c|c|c|c|c|c|c|c|c|c|c|c|}
\hline Attributes & \multicolumn{3}{|c|}{ Venture capitalist 1} & \multicolumn{3}{c|}{ Venture capitalist 2} & \multicolumn{3}{c|}{ Venture capitalist 3} \\
\cline { 2 - 13 } & $c_{1}$ & $c_{2}$ & $c_{3}$ & $c_{4}$ & $c_{1}$ & $c_{2}$ & $c_{3}$ & $c_{4}$ & $c_{1}$ & $c_{2}$ & $c_{3}$ & $c_{4}$ \\
\hline$D_{j}^{k}\left(H_{2 j}^{k}, H_{3 j}^{k}\right)$ & -0.286 & -0.333 & -0.143 & -0.286 & -0.214 & -0.286 & -0.143 & -0.190 & -0.071 & -0.048 & -0.143 & -0.143 \\
\hline$D_{j}^{k}\left(H_{2 j}^{k}, H_{4 j}^{k}\right)$ & 0 & -0.143 & 0.048 & -0.048 & -0.071 & -0.048 & 0 & 0 & 0 & 0.143 & 0 & -0.143 \\
\hline$D_{j}^{k}\left(H_{3 j}^{k}, H_{1 j}^{k}\right)$ & 0.143 & 0 & 0.071 & 0.143 & 0.143 & 0 & 0.095 & 0.048 & -0.071 & 0 & 0.071 & -0.143 \\
\hline$D_{j}^{k}\left(H_{3 j}^{k}, H_{2 j}^{k}\right)$ & 0.286 & 0.333 & 0.143 & 0.286 & 0.214 & 0.286 & 0.143 & 0.190 & 0.071 & 0.048 & 0.143 & 0.143 \\
\hline$D_{j}^{k}\left(H_{3 j}^{k}, H_{4 j}^{k}\right)$ & 0.286 & 0.190 & 0.214 & 0.238 & 0.143 & 0.238 & 0.143 & 0.190 & 0.071 & 0.190 & 0.143 & 0 \\
\hline$D_{j}^{k}\left(H_{4 j}^{k}, H_{1 j}^{k}\right)$ & -0.143 & -0.190 & -0.143 & -0.095 & 0.095 & -0.238 & -0.048 & -0.143 & -0.143 & -0.190 & -0.095 & -0.143 \\
\hline$D_{j}^{k}\left(H_{4 j}^{k}, H_{2 j}^{k}\right)$ & 0 & 0.143 & -0.048 & 0.048 & 0.071 & 0.048 & 0 & 0 & 0 & -0.143 & 0 & 0.143 \\
\hline$D_{j}^{k}\left(H_{4 j}^{k}, H_{3 j}^{k}\right)$ & -0.286 & -0.190 & -0.214 & -0.238 & -0.143 & -0.238 & -0.143 & -0.190 & -0.071 & -0.190 & -0.143 & 0 \\
\hline
\end{tabular}

$D_{j}^{k}\left(H_{i j}^{k}, H_{i j}^{k}\right)=0(i \in N)$ are not presented in Table 2, by the way, for $i \in N, P_{j}^{k}\left(x_{i}, x_{i}\right)=0$ and $G_{j}^{k}\left(x_{i}, x_{i}\right)=0$ are not exhibited in the following tables as well.

Step 4. The preferences between the start-up firms under each attribute are listed in Table 3 on the basis of Eqs (11) and (12).

Table 3. Preference values between the start-up firms

\begin{tabular}{|c|c|c|c|c|c|c|c|c|c|c|c|c|}
\hline Attributes & \multicolumn{3}{|c|}{ Venture capitalist 1} & \multicolumn{3}{c|}{ Venture capitalist 2} & \multicolumn{3}{c|}{ Venture capitalist 3} \\
\cline { 2 - 14 } & $c_{1}$ & $c_{2}$ & $c_{3}$ & $c_{4}$ & $c_{1}$ & $c_{2}$ & $c_{3}$ & $c_{4}$ & $c_{1}$ & $c_{2}$ & $c_{3}$ & $c_{4}$ \\
\hline$P_{j}^{k}\left(x_{1}, x_{2}\right)$ & 0.714 & 1 & 0.381 & 0.238 & 0.238 & 0.952 & 0.190 & 0.238 & 0.714 & 0.159 & 0.381 & 0.476 \\
\hline$P_{j}^{k}\left(x_{1}, x_{3}\right)$ & -0.714 & 0 & -0.286 & -0.238 & -0.714 & 0 & -0.381 & -0.079 & 0.357 & 0 & -0.286 & 0.238 \\
\hline$P_{j}^{k}\left(x_{1}, x_{4}\right)$ & 0.714 & 0.635 & 0.571 & 0.159 & -0.476 & 0.794 & 0.190 & 0.238 & 0.714 & 0.635 & 0.381 & 0.238 \\
\hline$P_{j}^{k}\left(x_{2}, x_{1}\right)$ & -0.714 & -1 & -0.381 & -0.238 & -0.238 & -0.952 & -0.190 & -0.238 & -0.714 & -0.159 & -0.381 & -0.476 \\
\hline$P_{j}^{k}\left(x_{2}, x_{3}\right)$ & -1 & -1 & -0.571 & -0.476 & -1 & -0.952 & -0.571 & -0.317 & -0.357 & -0.159 & -0.571 & -0.238 \\
\hline$P_{j}^{k}\left(x_{2}, x_{4}\right)$ & 0 & -0.476 & 0.190 & -0.079 & -0.357 & -0.159 & 0 & 0 & 0 & 0.476 & 0 & -0.238 \\
\hline$P_{j}^{k}\left(x_{3}, x_{1}\right)$ & 0.714 & 0 & 0.286 & 0.238 & 0.714 & 0 & 0.381 & 0.079 & -0.357 & 0 & 0.286 & -0.238 \\
\hline$P_{j}^{k}\left(x_{3}, x_{2}\right)$ & 1 & 1 & 0.571 & 0.476 & 1 & 0.952 & 0.571 & 0.317 & 0.357 & 0.159 & 0.571 & 0.238 \\
\hline$P_{j}^{k}\left(x_{3}, x_{4}\right)$ & 1 & 0.635 & 0.857 & 0.397 & 0.714 & 0.794 & 0.571 & 0.317 & 0.357 & 0.635 & 0.571 & 0 \\
\hline$P_{j}^{k}\left(x_{4}, x_{1}\right)$ & -0.714 & -0.635 & -0.571 & -0.159 & 0.476 & -0.794 & -0.190 & -0.238 & -0.714 & -0.635 & -0.381 & -0.238 \\
\hline$P_{j}^{k}\left(x_{4}, x_{2}\right)$ & 0 & 0.476 & -0.190 & 0.079 & 0.357 & 0.159 & 0 & 0 & 0 & -0.476 & 0 & 0.238 \\
\hline$P_{j}^{k}\left(x_{4}, x_{3}\right)$ & -1 & -0.635 & -0.857 & -0.397 & -0.714 & -0.794 & -0.571 & -0.317 & -0.357 & -0.635 & -0.571 & 0 \\
\hline
\end{tabular}


Step 5. The global preference indices between the start-up firms are acquired in Table 4:

Table 4. Global preference indices obtained by the G-PROMETHEE

\begin{tabular}{|c|c|c|c|c|c|c|c|}
\hline VCs & $z_{1}$ & $z_{2}$ & $z_{3}$ & & VCs & & \\
\hline$G^{k}\left(x_{1}, x_{2}\right)$ & 2.333 & 1.619 & 1.730 & $G^{k}\left(x_{3}, x_{1}\right)$ & 1.238 & 1.175 & -0.310 \\
\hline$G^{k}\left(x_{1}, x_{3}\right)$ & -1.238 & -1.175 & 0.310 & $G^{k}\left(x_{3}, x_{2}\right)$ & 3.048 & 2.841 & 1.325 \\
\hline$G^{k}\left(x_{1}, x_{4}\right)$ & 2.079 & 0.746 & 1.968 & $G^{k}\left(x_{3}, x_{4}\right)$ & 2.889 & 2.397 & 1.563 \\
\hline$G^{k}\left(x_{2}, x_{1}\right)$ & -2.333 & -1.619 & -1.730 & $G^{k}\left(x_{4}, x_{1}\right)$ & -2.079 & -0.746 & -1.968 \\
\hline$G^{k}\left(x_{2}, x_{3}\right)$ & -3.048 & -2.841 & -1.325 & $G^{k}\left(x_{4}, x_{2}\right)$ & 0.365 & 0.516 & -0.238 \\
\hline$G^{k}\left(x_{2}, x_{4}\right)$ & -0.365 & -0.516 & 0.238 & $G^{k}\left(x_{4}, x_{3}\right)$ & -2.889 & -2.397 & -1.563 \\
\hline
\end{tabular}

Step 6. The positive and negative outranking flows are derived by using Eqs (14) and (15).

Step 7. The net outranking flows are calculated according to Eqs (16) and (17) and the results are showing in Table 5:

Table 5. Net out ranking flows

\begin{tabular}{|c|c|c|c|}
\hline Net out ranking flow & $z_{1}$ & $z_{2}$ & $z_{3}$ \\
\hline$\phi^{k}\left(x_{1}^{k}\right)$ & 2.116 & 0.794 & 2.672 \\
\hline$\phi^{k}\left(x_{2}^{k}\right)$ & -3.831 & -3.317 & -1.878 \\
\hline$\phi^{k}\left(x_{3}^{k}\right)$ & 4.783 & 4.275 & 1.720 \\
\hline$\phi^{k}\left(x_{4}^{k}\right)$ & -3.069 & -1.751 & -2.513 \\
\hline
\end{tabular}

Step 8. The weights of the VCs and the ranking results are presented in Table 6 and Table 7 correspondingly.

Table 6. Weights of the VCs

\begin{tabular}{|c|c|c|c|}
\hline Weight & $z_{1}$ & $z_{2}$ & $z_{3}$ \\
\hline$\pi_{k}$ & 0.353 & 0.556 & 0.091 \\
\hline
\end{tabular}

Table 7. Net out ranking flow

\begin{tabular}{|l|c|c|c|c|}
\hline \multicolumn{1}{|c|}{ Alternatives } & $x_{1}$ & $x_{2}$ & $x_{3}$ & $x_{4}$ \\
\hline The net out ranking flows & 1.432 & -3.367 & 4.221 & -2.285 \\
\hline The ranking results & 2 & 4 & 1 & 3 \\
\hline
\end{tabular}


In Table 7, it is shown that $\psi\left(x_{3}\right)>\psi\left(x_{1}\right)>\psi\left(x_{4}\right)>\psi\left(x_{2}\right)$, that is, $x_{3} \succ x_{1} \succ x_{4} \succ x_{2}$. Step 9. End.

\subsection{Results of the GP-PROMETHEE with hesitant fuzzy linguistic information}

In Section 4.2, the ranking results of the G-PROMETHEE with hesitant fuzzy linguistic information are presented in Table 3. In this part, the GP-PROMETHEE with hesitant fuzzy linguistic information will be used to analyze the same illustrative example above for the sake of comparing the advantages of the two methods easily.

Step 1. For ease of comparisons, the original evaluation information under hesitant fuzzy linguistic circumstance is shown in Table 1.

Step 2. Considering the situation in Step 1, the standardized evaluation matrix follows Step 2 in Section 4.2 as well.

Step 3. The deviations of the prospect values are calculated according to Eqs (23)-(25) and the results are listed in Table 8.

Table 8. Deviations of the prospect values from VCs

\begin{tabular}{|c|c|c|c|c|c|c|c|c|c|c|c|c|}
\hline \multirow{2}{*}{ Deviations } & \multicolumn{3}{|c|}{ Venture capitalist 1} & \multicolumn{3}{c|}{ Venture capitalist 2} & \multicolumn{4}{c|}{ enture capitalist 3} \\
\hline & $c_{1}$ & $c_{2}$ & $c_{3}$ & $c_{4}$ & $c_{1}$ & $c_{2}$ & $c_{3}$ & $c_{4}$ & $c_{1}$ & $c_{2}$ & $c_{3}$ & $c_{4}$ \\
\hline$V_{j}^{k}\left(x_{1}, x_{2}\right)$ & 0.079 & 0.066 & 0.035 & 0.041 & 0.030 & 0.057 & 0.019 & 0.041 & 0.079 & 0.012 & 0.035 & 0.075 \\
\hline$V_{j}^{k}\left(x_{1}, x_{3}\right)$ & -0.193 & 0 & -0.062 & -0.088 & -0.193 & 0 & -0.079 & -0.033 & 0.043 & 0 & -0.063 & 0.041 \\
\hline$V_{j}^{k}\left(x_{1}, x_{4}\right)$ & 0.079 & 0.040 & 0.050 & 0.029 & -0.135 & 0.049 & 0.019 & 0.041 & 0.079 & 0.040 & 0.035 & 0.041 \\
\hline$V_{j}^{k}\left(x_{2}, x_{1}\right)$ & -0.193 & -0.133 & -0.079 & -0.088 & -0.073 & -0.116 & -0.043 & -0.088 & -0.193 & -0.024 & -0.079 & -0.161 \\
\hline$V_{j}^{k}\left(x_{2}, x_{3}\right)$ & -0.356 & -0.133 & -0.113 & -0.161 & -0.276 & -0.116 & -0.113 & -0.113 & -0.105 & -0.024 & -0.113 & -0.088 \\
\hline$V_{j}^{k}\left(x_{2}, x_{4}\right)$ & 0 & -0.063 & 0.019 & -0.033 & -0.105 & -0.024 & 0 & 0 & 0 & 0.031 & 0 & -0.088 \\
\hline$V_{j}^{k}\left(x_{3}, x_{1}\right)$ & 0.079 & 0 & 0.027 & 0.041 & 0.079 & 0 & 0.035 & 0.016 & -0.105 & 0 & 0.027 & -0.088 \\
\hline$V_{j}^{k}\left(x_{3}, x_{2}\right)$ & 0.146 & 0.066 & 0.050 & 0.075 & 0.113 & 0.057 & 0.050 & 0.053 & 0.043 & 0.012 & 0.050 & 0.041 \\
\hline$V_{j}^{k}\left(x_{3}, x_{4}\right)$ & 0.146 & 0.040 & 0.072 & 0.064 & 0.079 & 0.049 & 0.050 & 0.053 & 0.043 & 0.040 & 0.050 & 0 \\
\hline$V_{j}^{k}\left(x_{4}, x_{1}\right)$ & -0.193 & -0.081 & -0.113 & -0.061 & 0.055 & -0.099 & -0.043 & -0.088 & -0.193 & -0.081 & -0.079 & -0.088 \\
\hline$V_{j}^{k}\left(x_{4}, x_{2}\right)$ & 0 & 0.031 & -0.043 & 0.016 & 0.043 & 0.012 & 0 & 0 & 0 & -0.063 & 0 & 0.041 \\
\hline$V_{j}^{k}\left(x_{4}, x_{3}\right)$ & -0.356 & -0.081 & -0.162 & -0.137 & -0.193 & -0.099 & -0.113 & -0.113 & -0.105 & -0.081 & -0.113 & 0 \\
\hline
\end{tabular}

$V_{j}^{k}\left(x_{i}, x_{i}\right)=0(i \in N)$ are not shown in the above table, and similarly, all $P_{j}^{k}\left(x_{i}, x_{i}\right)=0$ and $G_{j}^{k}\left(x_{i}, x_{i}\right)=0(i \in N)$ are not listed in the following tables.

Step 4. The VCs' preferences between the bike-share firms under all attributes will be worked out according to Eqs (12) and (26). Please see the results in Table 9. 
Table 9. Prospect preference values from VCs

\begin{tabular}{|c|c|c|c|c|c|c|c|c|c|c|c|c|}
\hline \multirow{2}{*}{ Attributes } & \multicolumn{3}{|c|}{ Venture capitalist 1 } & \multicolumn{3}{c|}{ Venture capitalist 2} & \multicolumn{3}{c|}{ Venture capitalist 3 } \\
\cline { 2 - 12 } & $c_{1}$ & $c_{2}$ & $c_{3}$ & $c_{4}$ & $c_{1}$ & $c_{2}$ & $c_{3}$ & $c_{4}$ & $c_{1}$ & $c_{2}$ & $c_{3}$ & $c_{4}$ \\
\hline$P_{j}^{k}\left(x_{1}, x_{2}\right)$ & 0.396 & 0.219 & 0.141 & 0.068 & 0.151 & 0.191 & 0.077 & 0.068 & 0.396 & 0.040 & 0.141 & 0.125 \\
\hline$P_{j}^{k}\left(x_{1}, x_{3}\right)$ & -0.966 & 0 & -0.246 & -0.146 & -0.966 & 0 & -0.317 & -0.056 & 0.215 & 0 & -0.246 & 0.068 \\
\hline$P_{j}^{k}\left(x_{1}, x_{4}\right)$ & 0.396 & 0.134 & 0.201 & 0.048 & -0.676 & 0.163 & 0.077 & 0.068 & 0.396 & 0.134 & 0.141 & 0.068 \\
\hline$P_{j}^{k}\left(x_{2}, x_{1}\right)$ & -0.966 & -0.443 & -0.317 & -0.146 & -0.367 & -0.387 & -0.172 & -0.146 & -0.966 & -0.080 & -0.317 & -0.269 \\
\hline$P_{j}^{k}\left(x_{2}, x_{3}\right)$ & -1 & -0.443 & -0.454 & -0.269 & -1 & -0.387 & -0.454 & -0.188 & -0.525 & -0.080 & -0.454 & -0.146 \\
\hline$P_{j}^{k}\left(x_{2}, x_{4}\right)$ & 0 & -0.210 & 0.077 & -0.056 & -0.525 & -0.080 & 0 & 0 & 0 & 0.104 & 0 & -0.146 \\
\hline$P_{j}^{k}\left(x_{3}, x_{1}\right)$ & 0.396 & 0 & 0.109 & 0.068 & 0.396 & 0 & 0.141 & 0.026 & -0.525 & 0 & 0.109 & -0.146 \\
\hline$P_{j}^{k}\left(x_{3}, x_{2}\right)$ & 0.729 & 0.219 & 0.201 & 0.125 & 0.566 & 0.191 & 0.201 & 0.088 & 0.215 & 0.040 & 0.201 & 0.068 \\
\hline$P_{j}^{k}\left(x_{3}, x_{4}\right)$ & 0.729 & 0.134 & 0.288 & 0.107 & 0.396 & 0.163 & 0.201 & 0.088 & 0.215 & 0.134 & 0.201 & 0 \\
\hline$P_{j}^{k}\left(x_{4}, x_{1}\right)$ & -0.966 & -0.271 & -0.454 & -0.102 & 0.277 & -0.330 & -0.172 & -0.146 & -0.966 & -0.271 & -0.317 & -0.146 \\
\hline$P_{j}^{k}\left(x_{4}, x_{2}\right)$ & 0 & 0.104 & -0.172 & 0.026 & 0.215 & 0.040 & 0 & 0 & 0 & -0.210 & 0 & 0.068 \\
\hline$P_{j}^{k}\left(x_{4}, x_{3}\right)$ & -1 & -0.271 & -0.648 & -0.229 & -0.966 & -0.330 & -0.454 & -0.188 & -0.525 & -0.271 & -0.454 & 0 \\
\hline
\end{tabular}

Step 5. The global preference indexes of the VCs will be presented in Table 10 based on Eq. (27).

Table 10. Global preference index

\begin{tabular}{|c|c|c|c|r|c|c|c|}
\hline VCs & $z_{1}$ & $z_{2}$ & $z_{3}$ & & VCs & & \\
\hline
\end{tabular}

Step 6. The positive outranking flow and the negative outranking flow of the VCs are determined by Eqs (14) and (15) correspondingly.

Step 7. Table 11 gives the net outranking flow from VCs based on Eq. (16). 
Table 11. Net out ranking flow

\begin{tabular}{|c|c|c|c|}
\hline Net out ranking flow & $z_{1}$ & $z_{2}$ & $z_{3}$ \\
\hline$\phi^{k}\left(x_{1}^{k}\right)$ & 1.112 & -0.113 & 1.791 \\
\hline$\phi^{k}\left(x_{2}^{k}\right)$ & -2.094 & -1.831 & -1.321 \\
\hline$\phi^{k}\left(x_{3}^{k}\right)$ & 2.926 & 2.587 & 0.977 \\
\hline$\phi^{k}\left(x_{4}^{k}\right)$ & -1.943 & -0.643 & -1.447 \\
\hline
\end{tabular}

Step 8. Table 12 exhibits the net outranking flow of each venture capitalist for all start-up firms.

Table 12. Net out ranking flow

\begin{tabular}{|l|c|c|c|c|}
\hline \multicolumn{1}{|c|}{ Alternatives } & $x_{1}$ & $x_{2}$ & $x_{3}$ & $x_{4}$ \\
\hline The net out ranking flow & 0.493 & -1.877 & 2.559 & -1.175 \\
\hline The ranking results & 2 & 4 & 1 & 3 \\
\hline
\end{tabular}

From Table 12, it is known that $\psi\left(x_{3}\right)>\psi\left(x_{1}\right)>\psi\left(x_{4}\right)>\psi\left(x_{2}\right)$. Thus, $x_{3} \succ x_{1} \succ x_{4} \succ x_{2}$, in other words, $x_{3}$ is considered as the best start-up firm among the four, whereas, $x_{2}$ is the worst one.

Step 9. End.

\subsection{Results of TODIM with hesitant fuzzy linguistic information}

The GP-PROMETHEE is established to simulate the psychological states of VCs through net outranking flow, however, the TODIM is another outranking method developed on PT to consider the behavioral decision-making of VCs. Therefore, in this section, the results of the TODIM with hesitant fuzzy linguistic information (Wei et al., 2015; L. Wang, Y. M. Wang, Rodríguez, \& Martínez, 2017a) will be calculated, and it is used to show the better results of the GP-PROMETHEE from the comparison of the TODIM and the GP-PROMETHEE.

Step 1. Obtain the decision information from experts as shown by Steps 1 and 2 in Section 4.2.

Step 2. Calculate the relative weight $\omega_{j r}$ according to the criteria weights in Section 4.1.

$$
\omega_{j r}=\frac{\omega_{j}}{\omega_{r}}
$$

where $r, j \in M, \omega_{r}=\max \left(\omega_{j} \mid j \in M\right)$ and $c_{r}$ is called a reference criterion. Then, $\omega_{1 r}=1$, $\omega_{2 r}=0.16, \omega_{3 r}=0.43, \omega_{4 r}=0.28$.

Step 3. Determine the $k$ th venture capitalist's dominance of the project $x_{a}$ over $x_{b}(a, b \in$ $N)$ : 


$$
\psi^{k}\left(x_{a}, x_{b}\right)=\sum_{j=1}^{m} \varphi_{j}^{k}\left(x_{a}, x_{b}\right)
$$

where

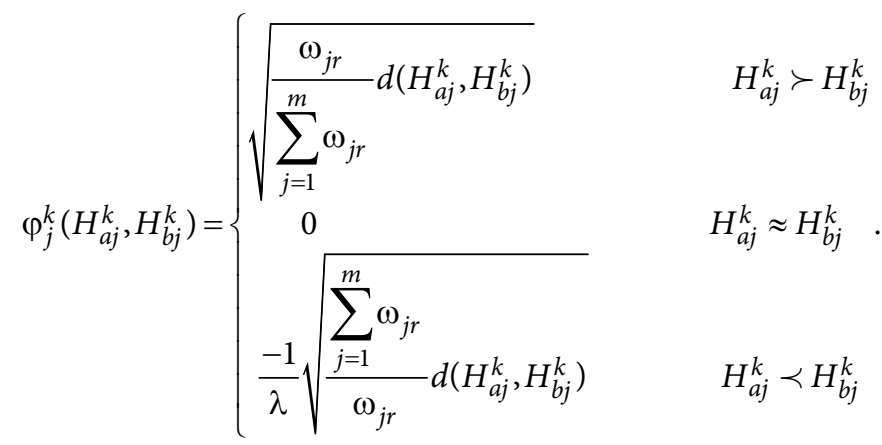

The parameter $\lambda$ is the attenuation factor of the losses and it is the same with in the GPPROMETHEE. Then, the results are listed in Table 13.

Table 13. Dominance among projects from each venture capitalist

\begin{tabular}{|c|c|c|c|c|c|c|c|}
\hline VCs & $z_{1}$ & $z_{2}$ & $z_{3}$ & VCs & $z_{1}$ & $z_{2}$ & $z_{3}$ \\
\hline$\psi^{k}\left(x_{1}, x_{2}\right)$ & 0.740 & 0.567 & 0.695 & $\psi^{k}\left(x_{3}, x_{1}\right)$ & 0.551 & 0.509 & -0.469 \\
\hline$\psi^{k}\left(x_{1}, x_{3}\right)$ & -0.913 & -0.767 & 0.094 & $\psi^{k}\left(x_{3}, x_{2}\right)$ & 0.948 & 0.845 & 0.587 \\
\hline$\psi^{k}\left(x_{1}, x_{4}\right)$ & 0.705 & 0.206 & 0.698 & $\psi^{k}\left(x_{3}, x_{4}\right)$ & 0.929 & 0.769 & 0.505 \\
\hline$\psi^{k}\left(x_{2}, x_{1}\right)$ & -1.826 & -1.580 & -1.462 & $\psi^{k}\left(x_{4}, x_{1}\right)$ & -1.597 & -1.151 & -1.612 \\
\hline$\psi^{k}\left(x_{2}, x_{3}\right)$ & -2.166 & -1.944 & -1.279 & $\psi^{k}\left(x_{4}, x_{2}\right)$ & -0.007 & 0.259 & -0.427 \\
\hline$\psi^{k}\left(x_{2}, x_{4}\right)$ & -0.719 & -0.493 & -0.324 & $\psi^{k}\left(x_{4}, x_{3}\right)$ & -1.977 & -1.822 & -1.174 \\
\hline
\end{tabular}

Step 4. Aggregate all the VCs' dominances of the project $x_{a}$ over $x_{b}(a, b \in N)$ :

$$
\psi\left(x_{a}, x_{b}\right)=\sum_{k=1}^{z} \pi_{k} \psi^{k}\left(x_{a}, x_{b}\right) .
$$

Step 5. Identify the overall value of the project $x_{i^{\prime}}$ :

$$
\Omega\left(x_{i^{\prime}}\right)=\frac{\sum_{b=1}^{n} \psi\left(x_{i^{\prime}}, x_{b}\right)-\min _{i}\left\{\sum_{b=1}^{n} \psi\left(x_{i^{\prime}}, x_{b}\right)\right\}}{\max _{i}\left\{\sum_{b=1}^{n} \psi\left(x_{i^{\prime}}, x_{b}\right)\right\}-\min _{i}\left\{\sum_{b=1}^{n} \psi\left(x_{i^{\prime}}, x_{b}\right)\right\}} \quad i^{\prime} \in N
$$


Then, $\Omega\left(x_{1}\right)=0.718, \Omega\left(x_{2}\right)=0, \Omega\left(x_{3}\right)=1, \Omega\left(x_{4}\right)=0.177$.

Step 6. Rank the overall value $\Omega\left(x_{i^{\prime}}\right), i^{\prime} \in N . x_{i^{\prime}}$ will be the promising project if $\max \left(\Omega\left(x_{i^{\prime}}\right), i^{\prime} \in N\right)$. According to the results in Step 5, $x_{3} \succ x_{1} \succ x_{4} \succ x_{2}$.

\subsection{Comparative analysis}

In this section, the comparative analysis will be conducted. On the one hand, it is necessary to analyze the feasibility and advantage of the proposed methods between the G-PROMETHEE and the GP-PROMETHEE. On the other hand, in order to explore the advantage of the GPPROMETHEE in simulating the VCs' behaviors, it is necessary to compare the applicability between the existing TODIM and the proposed GP-PROMETHEE.

\subsubsection{Comparison of the G-PROMETHEE and the GP-PROMETHEE}

As Table 14 shows, the ranking results of the two methods are identical in this case. Both of them consider $x_{3}$ as the best bike-share firm to be invested, and consider $x_{2}$ as the worst one. But the values of the net outranking flow are different under those two methods.

Table 14. Net outranking flow of the two methods

\begin{tabular}{|c|c|c|c|c|}
\hline Methods $\quad$ Net out ranking flow & $\psi\left(x_{1}\right)$ & $\psi\left(x_{2}\right)$ & $\psi\left(x_{3}\right)$ & $\psi\left(x_{4}\right)$ \\
\hline The G-PROMETHEE & 1.432 & -3.367 & 4.221 & -2.285 \\
\hline The GP-PROMETHEE & 0.493 & -1.877 & 2.559 & -1.175 \\
\hline
\end{tabular}

As has been mentioned before, $q$ and $\bar{q}$ are the parameters of the proposed methods, it is necessary to discuss the effect of those parameters on the ranking results. Hence, Table 15 shows the ranking results of the proposed methods with different parameters:

Table 15. The ranking results

\begin{tabular}{|l|c|c|}
\hline \multicolumn{1}{|c|}{ Methods } & The G-PROMETHEE & The GP-PROMETHEE \\
\hline Values of parameters & $x_{3} \succ x_{1} \succ x_{4} \succ x_{2}$ & $x_{3} \succ x_{1} \succ x_{4} \succ x_{2}$ \\
$\underline{q}_{1}=0, \underline{q}_{2}=0, \underline{q}_{3}=0, \underline{q}_{4}=0$ & $x_{1}=0.2, \bar{q}_{2}=0.3, \bar{q}_{3}=0.25, \bar{q}_{4}=0.6$ & $x_{3} \succ x_{1} \succ x_{4} \succ x_{2}$ \\
\hline$\underline{q}_{1}=0.05, \underline{q}_{2}=0.05, \underline{q}_{3}=0.05, \underline{q}_{4}=0.05$ & & \\
\hline $\bar{q}_{1}=0.2, \bar{q}_{2}=0.3, \bar{q}_{3}=0.25, \bar{q}_{4}=0.6$ & $x_{3} \succ x_{1} \succ x_{4} \succ x_{2}$ & $x_{3} \succ x_{1} \succ x_{4} \succ x_{2}$ \\
\hline$\underline{q}_{1}=0.1, \underline{q}_{2}=0.1, \underline{q}_{3}=0.1, \underline{q}_{4}=0.1$ & \\
$\bar{q}_{1}=0.2, \bar{q}_{2}=0.3, \bar{q}_{3}=0.25, \bar{q}_{4}=0.6$ & $x_{3} \succ x_{1} \succ x_{4} \succ x_{2}$ & $x_{3} \succ x_{1} \succ x_{4} \succ x_{2}$ \\
\hline$\underline{q}_{1}=0.15, \underline{q}_{2}=0.15, \underline{q}_{3}=0.15, \underline{q}_{4}=0.15$ & \\
$\bar{q}_{1}=0.2, \bar{q}_{2}=0.3, \bar{q}_{3}=0.25, \bar{q}_{4}=0.6$ & & \\
\hline
\end{tabular}


End of Table 15

\begin{tabular}{|l|c|c|}
\hline \multicolumn{1}{|c|}{ Methods } & The G-PROMETHEE & The GP-PROMETHEE \\
\hline Values of parameters & $x_{3} \succ x_{1} \succ x_{4} \succ x_{2}$ & $x_{3} \succ x_{1} \succ x_{4} \succ x_{2}$ \\
\hline$\underline{q}_{1}=0.2, \underline{q}_{2}=0.2, \underline{q}_{3}=0.2, \underline{q}_{4}=0.2$ & $x_{1} \succ x_{1} \succ x_{4} \succ x_{2}$ & $x_{3} \succ x_{1} \succ x_{4} \succ x_{2}$ \\
\hline $\bar{q}_{1}=0.2, \bar{q}_{2}=0.3, \bar{q}_{3}=0.25, \bar{q}_{4}=0.6$ & $x_{3} \succ x_{1} \succ x_{4} \succ x_{2}$ & $x_{3} \succ x_{1} \succ x_{4} \succ x_{2}$ \\
$\underline{q}_{1}=0, \underline{q}_{2}=0, \underline{q}_{3}=0, \underline{q}_{4}=0$ & \\
\hline $\bar{q}_{1}=0.4, \bar{q}_{2}=0.75, \bar{q}_{3}=0.28, \bar{q}_{4}=0.32$ & $x_{3} \succ x_{1} \succ x_{4} \succ x_{2}$ & $x_{3} \succ x_{1} \succ x_{4} \succ x_{2}$ \\
\hline$\underline{q}_{1}=0.05, \underline{q}_{2}=0.05, \underline{q}_{3}=0.05, \underline{q}_{4}=0.05$ & & \\
\hline $\bar{q}_{1}=0.3, \bar{q}_{2}=0.78, \bar{q}_{3}=0.36, \bar{q}_{4}=0.4$ & $\underline{q}_{1}=0.27, \underline{q}_{2}=0.2, \underline{q}_{3}=0.4, \underline{q}_{4}=0.9$ & \\
\hline $\bar{q}_{1}=0.34, \bar{q}_{2}=0.68, \bar{q}_{3}=0.32, \bar{q}_{4}=0.45$ & & \\
\hline
\end{tabular}

It is easily recognized that the ranking results will not change under different parameters, which indicates the strong stability of the proposed methods.

The biggest difference between the GP-PROMETHEE and the G-PROMETHEE which are proposed in this paper lies in that the former one introduces the effect of behaviors of the VCs on the decision-making process. From the theoretical aspect, the GP-PROMETHEE is better than the G-PROMETHEE. For example, numerous laboratory experiments have shown that the different attitudes of the VCs are indeed reflected in the aspect of gains and losses (Mattos \& Garcia, 2011). Moreover, the VCs' attitude with respect to risk aversion for gains or risk seeking for loss is more prominent under the highly uncertain circumstance of the VC. This different attitude for gains or losses has been considered in the GP-PROMETHEE as the value function of Eq. (24), and it has been demonstrated in our interview as well. In addition, whatever the proposed methods are, the criteria weights affect the decision making via its structure and strength, whereas, the transformed probability adopted by VCs has been confirmed (Mattos \& Garcia, 2011). Therefore, the GP-PROMETHEE using the weighting function rather than the objective probability is better than the G-PROMETHEE. On account of the different risk attitudes for gains and losses, the weighting function enhances the negative value or the positive value of the net outranking flow respectively under the GP-PROMETHEE. To sum up, the GP-PROMETHEE that combines the ranking method (PROMETHEE) with the behavioral theory (the PT which includes the value function to simulate the risk attitudes of the VCs and the weighting function to portray the transformed probability perceived by the VCs) exhibits more advantages in selecting the promising startup firm.

\subsubsection{Comparison of the GP-PROMETHEE and the TODIM}

As is shown in the results of Sections 4.3 and 4.4, the rankings of the projects in those two methods, the GP-PROMETHEE and the TODIM, are the same. Then, in order to compare the two methods, we test the stability of them since there is the parameter $\lambda$ which has the same meaning. 
Table 16. Ranking results

\begin{tabular}{|c|c|c|}
\hline Methods & The TODIM & The GP-PROMETHEE \\
\hline$\lambda=2.25$ & $x_{3} \succ x_{1} \succ x_{4} \succ x_{2}$ & $x_{3} \succ x_{1} \succ x_{4} \succ x_{2}$ \\
\hline$\lambda=2.15$ & $x_{3} \succ x_{1} \succ x_{4} \succ x_{2}$ & $x_{3} \succ x_{1} \succ x_{4} \succ x_{2}$ \\
\hline$\lambda=2.05$ & $x_{3} \succ x_{1} \succ x_{4} \succ x_{2}$ & $x_{3} \succ x_{1} \succ x_{4} \succ x_{2}$ \\
\hline$\lambda=1.85$ & $x_{3} \succ x_{1} \succ x_{4} \succ x_{2}$ & $x_{3} \succ x_{1} \succ x_{4} \succ x_{2}$ \\
\hline$\lambda=1.79$ & $x_{3} \succ x_{1} \succ x_{4} \succ x_{2}$ & $x_{3} \succ x_{1} \succ x_{4} \succ x_{2}$ \\
\hline$\lambda=1.75$ & $x_{3} \succ x_{1} \succ x_{4} \succ x_{2}$ & $x_{3} \succ x_{1} \succ x_{4} \succ x_{2}$ \\
\hline$\lambda=1.55$ & $x_{3} \succ x_{1} \succ x_{4} \succ x_{2}$ & $x_{3} \succ x_{1} \succ x_{4} \succ x_{2}$ \\
\hline$\lambda=1.25$ & $x_{3} \succ x_{1} \succ x_{4} \succ x_{2}$ & $x_{3} \succ x_{1} \succ x_{4} \succ x_{2}$ \\
\hline
\end{tabular}

According to Table 16, when $\lambda$ changes, the ranking results of both GP-PROMETHEE and TODIM do not change. But the changes of the final value are different in those two methods. In TODIM, the overall value of the best or worst project has always been 1 or 0 , whereas, the overall values of the other projects change between 0 and 1 . The change of $\lambda$ only bring little changes of overall values of projects. In GP-PROMETHEE, the values of net outranking flow of projects are not limited within 0 and 1 . It is much easier for DMs to recognize the best project among the similar ones. Therefore, the GP-PROMETHEE is better than the TODIM under hesitant fuzzy linguistic circumstance.

The advantages of the GP-PROMETHEE than the G-PROMETHEE and the TODIM are comprehensively shown by the comparative analysis in Section 4.5. They are summarized as follows: (1) The GP-PROMETHEE considers both the VCs' risk attitudes such as risk seeking for losses and risk aversion for gains and the transformed probability, whereas, the other methods usually ignore the transformed probability. (2) Although there are parameters in the GP-PROMETHEE, it is stable and effective.

\section{Conclusions}

Because of the fact that the VCs do not always access all the start-up firms, ranking the available start-up firms and finding the promising one become a common means used by the VCs in decision-making. Therefore, the ranking method plays an important role in the decision-making process. Among the numerous ranking methods, the PROMETHEE is a prevalent one. While in the decision-making process, the evaluation information is always presented as the hesitant fuzzy linguistic form. Moreover, when the VCs give the evaluation information, they exhibit behavioral characteristics such as certainty effect, reflection effect, loss aversion, risk seeking, reference dependence and so on, which are reflected by the PT. In addition, group decision making can overcome the deficiency of individual decisionmaking and it becomes an inevitable trend. Considering all the factors above, the group decision-making methods under hesitant fuzzy linguistic circumstances, which are named 
as the G-PROMETHEE and the GP-PROMETHEE, have been constructed to assist the VCs' investment decision-making.

The PROMETHEE has been firstly extended under hesitant fuzzy linguistic circumstance in this paper. The advantages of it can be summarized as: (1) According to the illustrative example, the GP-PROMETHEE, which adopts the merit of both the PT and the hesitant fuzzy linguistic information to portray the behavioral characteristics of the VCs, is better than the G-PROMETHEE and the TODIM according to the comparative analysis. (2) The behavioral decision-making will be promoted through the construction of GP-PROMETHEE. It will accelerate the development of decision sciences and play a demonstrated role in the research of other behavioral decision-making fields, such as the combination of regret theory and MCDM methods. (3) The proposed methods have been applied to solve the decision-making problem of VCs and the applications of them has been enriched. (4) The way to solve VC problem is abundant by the proposed methods and the introduction of hesitant fuzzy linguistic information to VC field will advance the development of behavioral finance under fuzzy circumstance.

Although there are some advantages of this GP-PROMETHEE, the behavioral decisionmaking with heterogeneous information catches our attention because the heterogeneous information is a common phenomenon of group decision making. Much deeper research about this phenomenon will be presented in our future work.

\section{Acknowledgements}

The work was supported in part by the China National Natural Science Foundation (Nos. 71771155, 71571123).

\section{References}

Afful-Dadzie, E., Nabareseh, S., Oplatková, Z. K., \& Klimek, P. (2016). Using fuzzy PROMETHEE to select countries for developmental aid. In Y. Bi, S. Kapoor, \& R. Bhatia (Eds.), Intelligent systems and applications. Series studies in computational intelligence (Vol. 650, pp. 109-132). Cham: Springer International Publishing. https://doi.org/10.1007/978-3-319-33386-1_6

Afful-Dadzie, E., Oplatková, Z. K., \& Nabareseh, S. (2015). Selecting start-up businesses in a public venture capital financing using fuzzy PROMETHEE. Procedia Computer Science, 60(1), 63-72. https://doi.org/10.1016/j.procs.2015.08.105

Amaral, T. M., \& Costa, A. P. C. (2014). Improving decision-making and management of hospital resources: An application of the PROMETHEE II method in an Emergency Department. Operations Research for Health Care, 3(1), 1-6. https://doi.org/10.1016/j.orhc.2013.10.002

Aouni, B., Colapinto, C., \& Torre, D. L. (2014). A fuzzy goal programming model for venture capital investment decision making. Information Systems and Operational Research, 52(3), 138-146. https://doi.org/10.3138/infor.52.3.138

Avikal, S., Mishra, P. K., \& Jain, R. (2014). A Fuzzy AHP and PROMETHEE method-based heuristic for disassembly line balancing problems. International Journal of Production Research, 52(5), 13061317. https://doi.org/10.1080/00207543.2013.831999

Babic, Z., \& Plazibat, N. (1998). Ranking of enterprises based on multicriterial analysis. International Journal of Production Economics, 56-57(1-3), 29-35. https://doi.org/10.1016/S0925-5273(97)00133-3 
Brans, J. P. (1982). L'ingénièrie de la décision; Elaboration d'instruments d'aide à la décision. La méthode PROMETHEE. In R. Nadeau \& M. Landry (Eds.), Laide à la décision: nature, instruments et perspectives d'avenir (pp. 183-214). Québec, Canada: Presses de l'Université Laval.

Brans, J. P., \& Mareschal, B. (1992). Promethee V: MCDM problems with segmentation constraints. Information Systems and Operational Research, 30(2), 85-96.

https://doi.org/10.1080/03155986.1992.11732186

Brans, J. P., \& Mareschal, B. (1995). The PROMETHEE VI procedure: How to differentiate hard from soft multicriteria problems. Journal of Decision Systems, 4(3), 213-223. https://doi.org/10.1080/12460125.1995.10511652

Brans, J. P., \& Vincke, P. H. (1985). A preference ranking organization method. Management Science, 31(6), 647-656. https://doi.org/10.1287/mnsc.31.6.647

Brans, J. P., Vincke, P. H., \& Mareschal, B. (1986). How to select and how to rank projects: The Promethee method. European Journal of Operational Research, 24(2), 228-238. https://doi.org/10.1016/0377-2217(86)90044-5

Bouri, A., Martel, J. M., \& Chabchoub, H. (2002). A multi-criterion approach for selecting attractive portfolio. Journal of Multi-Criteria Decision Analysis, 11(4-5), 269-277. https://doi.org/10.1002/mcda.334

Gannon, R., Hogan, K. M., \& Olson, G. T. (2015). A multicriteria decision model for venture capital firms' evaluation of new technology business firms. In Applications of Management Science (pp. 27-50). Emerald Group Publishing Limited.

Chen, C. T., Hung, W. Z., \& Cheng, H. L. (2011a). Applying linguistic PROMETHEE method in investment portfolio decision-making. International Journal of Electronic Business Management, 9(2), 139-148.

Chen, C. T., Pai, P. F., \& Hung, W. Z. (2010). An integrated methodology using linguistic PROMETHEE and maximum deviation method for third-party logistics supplier selection. International Journal of Computational Intelligence Systems, 3(4), 438-451. https://doi.org/10.1080/18756891.2010.9727712

Chen, T. Y. (2014). A PROMETHEE-based outranking method for multiple criteria decision analysis with interval type-2 fuzzy sets. Soft Computing, 18(5), 923-940.

https://doi.org/10.1007/s00500-013-1109-4

Chen, T. Y. (2015). IVIF-PROMETHEE outranking methods for multiple criteria decision analysis based on interval-valued intuitionistic fuzzy sets. Fuzzy Optimization and Decision Making, 14(2), 173-198. https://doi.org/10.1007/s10700-014-9195-Z

Chen, Y. H., Wang, T. C., \& Wu, C. Y. (2011b). Strategic decisions using the fuzzy PROMETHEE for IS outsourcing. Expert Systems with Applications, 38(10), 13216-13222. https://doi.org/10.1016/j.eswa.2011.04.137

Colapinto, C., \& Torre, D. L. (2015). Multiple criteria decision making and goal programming for optimal venture capital investments and portfolio management (pp. 9-30). Switzerland: Springer International Publishing. https://doi.org/10.1007/978-3-319-21158-9_2

Delgado, M., Verdegay, J. L., \& Vila, M. A. (1993). On aggregation operations of linguistic labels. International Journal of Intelligent Systems, 8(3), 351-370. https://doi.org/10.1002/int.4550080303

Goumas, M., \& Lygerou, V. (2000). An extension of the PROMETHEE method for decision making in fuzzy environment: Ranking of alternative energy exploitation projects. European Journal of Operational Research, 123(3), 606-613. https://doi.org/10.1016/S0377-2217(99)00093-4

Govindan, K., Kadziński, M., \& Sivakumar, R. (2017). Application of a novel PROMETHEE-based method for construction of a group compromise ranking to prioritization of green suppliers in food supply chain. Omega, 71, 129-145. https://doi.org/10.1016/j.omega.2016.10.004

Gul, M., Celik, E., Gumus, A. T., \& Guneri, A. F. (2018). A fuzzy logic based promethee method for material selection problems. Beni-Suef University Journal of Basic and Applied Sciences, 7(1), 68-79. https://doi.org/10.1016/j.bjbas.2017.07.002 
Halouani, N., Chabchoub, H., \& Martel, J. M. (2009). PROMETHEE-MD-2T method for project selection. European Journal of Operational Research, 195(3), 841-849.

https://doi.org/10.1016/j.ejor.2007.11.016

Herrera, F., \& Martínez, L. (2000). A 2-tuple fuzzy linguistic representation model for computing with words. IEEE Transactions on Fuzzy Systems, 8(6), 746-752. https://doi.org/10.1109/91.890332

Jiménez, M. (2005). PROMETHEE: An extension through fuzzy mathematical programming. Journal of the Operational Research Society, 56(1), 119-122. https://doi.org/10.1057/palgrave.jors.2601828

Kabir, G., \& Sumi, R. S. (2014). Power substation location selection using fuzzy analytic hierarchy process and PROMETHEE: A case study from Bangladesh. Energy, 72(2), 717-730. https://doi.org/10.1016/j.energy.2014.05.098

Kahneman, D., \& Tversky, A. (1979). Prospect theory: An analysis of decision under risk. Econometrica, 47(2), 263-291. https://doi.org/10.2307/1914185

Kahraman, C., Onar, S. C., \& Oztaysi, B. (2015). Fuzzy multicriteria decision-making: A literature review. International Journal of Computational Intelligence Systems, 8(4), 637-666. https://doi.org/10.1080/18756891.2015.1046325

Kilic, H. S., Zaim, S., \& Delen, D. (2015). Selecting “The Best” ERP system for SMEs using a combination of ANP and PROMETHEE methods. Expert Systems with Applications, 42(5), 2343-2352. https://doi.org/10.1016/j.eswa.2014.10.034

Krishankumar, R., Ravichandran, K. S., \& Saeid, A. B. (2017). A new extension to PROMETHEE under intuitionistic fuzzy environment for solving supplier selection problem with linguistic preferences. Applied Soft Computing, 60, 564-576. https://doi.org/10.1016/j.asoc.2017.07.028

Lee, M. C., \& Chang, T. (2010). Linguistic variables and PROMETHEE method as tools in evaluation of quality of portal website service. International Journal of Research and Reviews in Computer Science, 1(3), 20-28.

Lerche, N., \& Geldermann, J. (2015). Integration of prospect theory into PROMETHEE-a case study concerning sustainable bioenergy concepts. International Journal of Multicriteria Decision Making, 5(4), 309-333. https://doi.org/10.1504/IJMCDM.2015.074085

Li, W. X., \& Li, B. Y. (2010). An extension of the Promethee II method based on generalized fuzzy numbers. Expert Systems with Applications, 37(7), 5314-5319. https://doi.org/10.1016/j.eswa.2010.01.004

Liao, B. Q., Wang, L. D., \& Liu, X. D. (2016). Possibility-based outranking comparison for PROMETHEE II with uncertain linguistic fuzzy variables. In International conference on oriental thinking and fuzzy logic. Switzerland: Springer International Publishing. https://doi.org/10.1007/978-3-319-30874-6_8

Liao, H. C., \& Xu, Z. S. (2014). Multi-criteria decision making with intuitionistic fuzzy PROMETHEE. Journal of Intelligent and Fuzzy Systems Applications in Engineering and Technology, 27(4), 17031717.

Liao, H. C., Xu, Z. S, \& Zeng, X. J. (2014). Distance and similarity measures for hesitant fuzzy linguistic term sets and their application in multi-criteria decision making. Information Sciences, 271, 125142. https://doi.org/10.1016/j.ins.2014.02.125

Liao, H. C., Xu, Z. S., \& Zeng, X. J. (2015). Hesitant fuzzy linguistic VIKOR method and its application in qualitative multiple criteria decision making. IEEE Transactions on Fuzzy Systems, 23(5), 13431355. https://doi.org/10.1109/TFUZZ.2014.2360556

Macharis, C., Brans, J. P., \& Mareschal, B. (1998). The GDSS PROMETHEE procedure: A PROMETHEE-GAIA based procedure for group decision support. Journal of Decision Systems, 7, 283-307.

Mahmoudi, A., Sadi-Nezhad, S., Makui, A., \& Vakili, M. R. (2016). An extension on PROMETHEE based on the typical hesitant fuzzy sets to solve multi-attribute decision-making problem. Kybernetes, 45(8), 1213-1231. https://doi.org/10.1108/K-10-2015-0271

Mateo, J. R. S. C. (2012). Fuzzy PROMETHEE. In Multi criteria analysis in the renewable energy industry. London: Springer. https://doi.org/10.1007/978-1-4471-2346-0_11 
Mattos, F., \& Garcia, P. (2011). Applications of behavioral finance to entrepreneurs and venture capitalists: Decision making under risk and uncertainty in futures and options markets. In Advances in entrepreneurial finance (pp. 191-205). New York: Springer.

Peko, I., Gjeldum, N., \& Bilić, B. (2018). Application of AHP, fuzzy AHP and PROMETHEE method in solving additive manufacturing process selection problem. Tehnički Vjesnik-Technical Gazette Scientific Professional Journal of Technical Faculties of the Josip Juraj Strossmayer University of Osijek, 25(2), 710-719.

Rodríguez, R. M., Martínez, L., \& Herrera, F. (2012). Hesitant fuzzy linguistic term sets for decision making. IEEE Transactions on Fuzzy Systems, 20(1), 109-119. https://doi.org/10.1109/TFUZZ.2011.2170076

Rodríguez, R. M., Martínez, L., \& Herrera, F. (2013). A group decision making model dealing with comparative linguistic expressions based on hesitant fuzzy linguistic term sets. Information Sciences, 241(12), 28-42. https://doi.org/10.1016/j.ins.2013.04.006

Rodríguez, R. M., Álvaro, L., \& Martínez, L. (2016). An overview on fuzzy modelling of complex linguistic preferences in decision making. International Journal of Computational Intelligence Systems, 9, 81-94. https://doi.org/10.1080/18756891.2016.1180821

Samanlioglu, F., \& Ayağ, Z. (2017). A fuzzy AHP-PROMETHEE II approach for evaluation of solar power plant location alternatives in Turkey. Journal of Intelligent and Fuzzy Systems, 33(2), 859-871. https://doi.org/10.3233/JIFS-162122

Samanlioglu, F., \& Ayağ, Z. (2016). Fuzzy ANP-based PROMETHEE II approach for evaluation of machine tool alternatives. Journal of Intelligent and Fuzzy Systems, 30(4), 2223-2235. https://doi.org/10.3233/IFS-151991

Shih, H. S., Chang, Y. T., \& Cheng, C. P. (2016). A generalized PROMETHEE III with risk preferences on losses and gains. International Journal of Information and Management Sciences, 27, 117-127.

Singh, A., Gupta, A., \& Mehra, A. (2016). Energy planning problems with interval-valued 2-tuple linguistic information. Operational Research, 1-28.

Treville, S. D., Petty, J. S., \& Wager, S. (2014). Economies of extremes: Lessons from venture-capital decision making. Journal of Operations Management, 32(6), 387-398. https://doi.org/10.1016/j.jom.2014.07.002

Tversky, A., \& Kahneman, D. (1992). Advances in prospect theory: Cumulative representation of uncertainty. Journal of Risk and Uncertainty, 5(4), 297-323. https://doi.org/10.1007/BF00122574

Wang, L., Labella, Á., Rodríguez, R. M., Wang, Y. M., \& Martínez, L. (2017). Managing non-homogeneous information and experts' psychological behavior in group emergency decision making. Symmetry, 9(10), 234. https://doi.org/10.3390/sym9100234

Wang, L., Wang, Y. M., \& Martínez, L. (2018). A group decision method based on prospect theory for emergency situations. Information Sciences, 418-419, 119-135.

https://doi.org/10.1016/j.ins.2017.07.037

Wang, L., Wang, Y. M., Rodríguez, R. M., \& Martínez, L. (2017). A hesitant fuzzy linguistic model for emergency decision making based on fuzzy TODIM method. In IEEE International Conference on Fuzzy Systems (pp. 1-6). Naples, Italy: IEEE. https://doi.org/10.1109/FUZZ-IEEE.2017.8015550

Wei, C., Rodríguez, R. M., \& Martínez, L. (2018). Uncertainty measures of extended hesitant fuzzy linguistic term sets. IEEE Transactions on Fuzzy Systems, 26(3), 1763-1768. https://doi.org/10.1109/TFUZZ.2017.2724023

Wei, C. P., Ren, Z. L., \& Rodríguez, R. M. (2015). A hesitant fuzzy linguistic TODIM Method based on a score function. International Journal of Computational Intelligence Systems, 8(4), 701-712. https://doi.org/10.1080/18756891.2015.1046329

Widyanto, H. A., \& Dalimunthe, Z. (2015). Evaluation criteria of venture capital firms investing on Indonesians' SME. New York: Social Science Electronic Publishing. 
Wiratno, S. E., Latiffianti, E., \& Wirawan, K. K. (2015). Selection of business funding proposals using analytic network process: A case study at a venture capital company. Procedia Manufacturing, 4, 237-243. https://doi.org/10.1016/j.promfg.2015.11.037

$\mathrm{Xu}, \mathrm{Z}$. S. (2005). Deviation measures of linguistic preference relations in group decision making. Omega, 33(3), 249-254. https://doi.org/10.1016/j.omega.2004.04.008

Yilmaz, B., \& Dağdeviren, M. (2011). A combined approach for equipment selection: F-PROMETHEE method and zero-one goal programming. Expert Systems with Applications, 38(9), 11641-11650. https://doi.org/10.1016/j.eswa.2011.03.043

Zadeh, L. A. (1975a). The concept of a linguistic variable and its applications to approximate reasoningI. Information Sciences, 8, 199-249. https://doi.org/10.1016/0020-0255(75)90036-5

Zadeh, L. A. (1975b). The concept of a linguistic variable and its applications to approximate reasoningII. Information Sciences, 8, 301-357. https://doi.org/10.1016/0020-0255(75)90046-8

Zadeh, L. A. (1975c). The concept of a linguistic variable and its applications to approximate reasoningIII. Information Sciences, 9, 43-80. https://doi.org/10.1016/0020-0255(75)90017-1

Zhang, X. B. (2012). Venture capital investment selection decision-making base on fuzzy theory. Physics Procedia, 25(22), 1369-1375. https://doi.org/10.1016/j.phpro.2012.03.248

Zhao, L. K. (2009). The study of complex multi-stage dynamic decision making in venture capital project based on piecewise function. In International Conference on Management Science and Engineering (pp. 107-111). Beijing, China.

Zhou, J. S. (2012). Research on appraisal model of venture capital investing project based on high-tech outcome transformation with uncertain linguistic information. Advances in Information Sciences and Service Sciences, 4(1), 224-229. https://doi.org/10.4156/aiss.vol4.issue1.29 